\title{
CLASSIFICATION OF SMOOTH FACTORIAL AFFINE SURFACES OF KODAIRA DIMENSION ZERO WITH TRIVIAL UNITS
}

\author{
TOMASZ PEŁKA AND PAWEŁ RAŹNY
}

\begin{abstract}
We give a corrected statement of [GM88, Theorem 2], which classifies smooth affine surfaces of Kodaira dimension zero, whose coordinate ring is factorial and has trivial units. Denote the class of such surfaces by $\mathcal{S}_{0}$. An infinite series of surfaces in $\mathcal{S}_{0}$, not listed in [GM88], was recently obtained by Freudenburg, Kojima and Nagamine [FKN19] as affine modifications of the plane. We complete their list to a series containing arbitrarily high-dimensional families of pairwise non-isomorphic surfaces in $\mathcal{S}_{0}$. Moreover, we classify them up to a diffeomorphism, showing that each occurs as an interior of a 4-manifold whose boundary is an exceptional surgery on a 2-bridge knot [BW01]. In particular, we show that $\mathcal{S}_{0}$ contains countably many pairwise non-homeomorphic surfaces.
\end{abstract}

We work with complex algebraic varieties.

\section{INTRODUCTION}

The structure of smooth affine surfaces of non-general type is essentially understood, see [Miy01, Chapter III]. It can be summarized in a following way, which parallels the classical Enriques-Kodaira classification of smooth projective surfaces. Let $S$ be a smooth affine surface and let $\kappa \in\{-\infty, 0,1,2\}$ be its Kodaira-Iitaka dimension. If $\kappa=-\infty$ then $S$ admits a $\mathbb{C}^{1}$-fibration [Miy01, III.1.3.2]. If $\kappa=1$ then $S$ has a (canonical) $\mathbb{C}^{*}$-fibration [Miy01, III.1.7.1]. The surfaces with $\kappa=0$ are peculiar, but understandable: they are obtained in a controlled way from some specific minimal models, see [Miy01, II.6.4].

Let $\mathcal{S}_{\kappa}$ be the class of smooth affine surfaces $S$ of Kodaira-Iitaka dimension $\kappa$, such that $\mathbb{C}[S]^{*}=\mathbb{C}^{*}$ and $\mathbb{C}[S]$ is factorial, i.e. all line bundles on $S$ are trivial. It is known [Miy01, III.2.2.1] that $S \in \mathcal{S}_{-\infty}$ if and only if $S \cong \mathbb{C}^{2}$. The above description of affine surfaces with $\kappa<2$ suggests that it is the class $\mathcal{S}_{0}$ where one could look for unusual examples.

In [GM88, Theorem 2], Gurjar and Miyanishi claimed that, up to an isomorphism, there are at most two surfaces in $\mathcal{S}_{0}$. This result, however, was recently proved wrong by Freudenburg, Kojima and Nagamine [FKN19], who constructed an infinite series of pairwise non-isomorphic surfaces in $\mathcal{S}_{0}$.

The aim of this article is to complete the description of $\mathcal{S}_{0}$ by proving the following theorem.

Theorem 1.1. Let $\mathcal{S}_{0}$ be the class of smooth affine surfaces $S$ such that the ring $\mathbb{C}[S]$ is factorial, $\mathbb{C}[S]^{*}=\mathbb{C}^{*}$ and the logarithmic Kodaira-Iitaka dimension of $S$ is zero. Then $S \in \mathcal{S}_{0}$ if and only if

$$
S \cong S_{p_{1}, p_{2}}:=\operatorname{Spec} \mathbb{C}\left[x_{1}, x_{2}\right]\left[\left(x_{2} x_{1}^{-\operatorname{deg} p_{1}}-p_{1}\left(x_{1}^{-1}\right)\right) x_{1}^{-1},\left(x_{1} x_{2}^{-\operatorname{deg} p_{2}}-p_{2}\left(x_{2}^{-1}\right)\right) x_{2}^{-1}\right]
$$

for some monic polynomials $p_{1}, p_{2} \in \mathbb{C}[t]$. Moreover, $S_{p_{1}, p_{2}} \neq S_{p_{1}^{\prime}, p_{2}^{\prime}}$ for $\left\{p_{1}, p_{2}\right\} \neq\left\{p_{1}^{\prime}, p_{2}^{\prime}\right\}$.

Remark 1.2. (a) The two surfaces constructed in [GM88, Theorem 2] are both isomorphic to $S_{1,1}$, see [FKN19, Theorem 4.4]. This surface was extensively studied in [KK11, §8].

(b) For $n \geqslant 1$, the surface $V_{n}=\operatorname{Spec} \mathbb{C}[x, y]\left[(x-1) /\left(x^{n} y-1\right)\right]$ constructed in [FKN19] is isomorphic to $S_{t^{n-1}, 1}$ via $\mathbb{C}\left[x_{1}, x_{2}\right]\left[\left(x_{2}-1\right) x_{1}^{-n},\left(x_{1}-1\right) x_{2}^{-1}\right] \ni\left(x_{1}, x_{2}\right) \mapsto\left(x, 1-x^{n} y\right) \in \mathbb{C}[x, y]\left[(x-1) /\left(x^{n} y-1\right)\right]$.

Construction 1.3. In the remaining part of the article we will use the following geometric construction of the surface $S_{p_{1}, p_{2}}$, or rather of its $\log$ smooth completion. Let $\left(x_{1}, x_{2}\right)$ be coordinates on $\mathbb{P}^{1} \times \mathbb{P}^{1}$. For $t \in \mathbb{P}^{1}$, $j \in\{1,2\}$ put $\ell_{j, t}=\left\{x_{j}=t\right\}$. Write $p_{1}(t)=t^{d-1}+a_{1} t^{d-2}+\cdots+a_{d-1}$. We perform a sequence of $d$ blowups over $(0,1)$, as follows. The exceptional curve of the first blowup is parametrized by $x_{2}^{\prime}=\left(x_{2}-1\right) x_{1}^{-1} \in \mathbb{P}^{1}$, where $x_{2}^{\prime}=\infty$ lies on the proper transform of $\ell_{1,0}$. We blow up the point $x_{2}^{\prime}=a_{1}$, and so on: after $m$-th blowup, the exceptional curve is parameterized by $x_{2}^{(m)}=x_{2} x_{1}^{-m}-x_{1}^{-m}-a_{1} x_{1}^{-m+1}-\cdots-a_{m-1}$, and we blow up the point with $x_{2}^{(m)}=a_{m}$. Next, we use $p_{2}$ to analogously define a sequence of $\operatorname{deg} p_{2}+1$ blowups over the preimage of $(1,0)$. We denote the resulting morphism by $\varphi: X \longrightarrow \mathbb{P}^{1} \times \mathbb{P}^{1}$, put $L_{j, t}:=\varphi_{*}^{-1} \ell_{j, t}$ and denote by $A_{1}, A_{2} \subseteq X$ the exceptional curves of the last blowup over $(0,1)$ and $(1,0)$, respectively. Eventually, we put $D=\varphi^{*}\left(\ell_{1, \infty}+\ell_{2, \infty}+\ell_{1,0}+\ell_{2,0}\right)_{\text {red }}-A_{1}-A_{2}$ and $S_{p_{1}, p_{2}}=X \backslash D$.

2010 Mathematics Subject Classification. Primary: 14R05; Secondary: 14J26, 57R65, 57M99.

Key words and phrases. affine surface, $\mathbb{C}^{*}$-fibration, log Minimal Model program, knot surgery, Kirby diagram. 


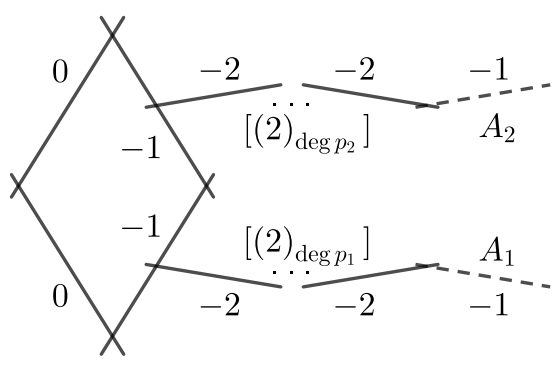

(A) $p_{1}, p_{2} \neq 1$

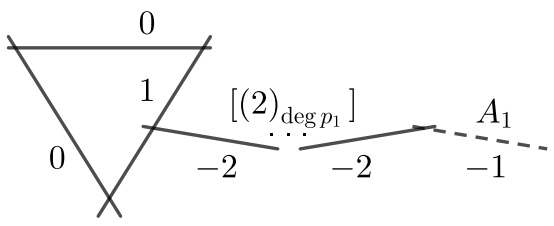

(в) $p_{1} \neq 1, p_{2}=1$

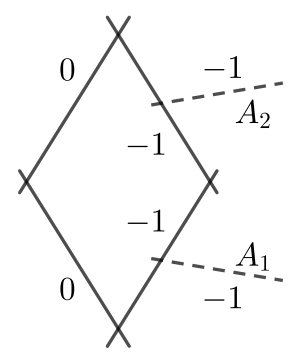

(c) $p_{1}=p_{2}=1$

FIgURE 1. Standard boundaries of the surfaces $S_{p_{1}, p_{2}}$ from Theorem 1.1.

Figure 1(a) shows the graph of $D+A_{1}+A_{2}$, where we use the following notation: each line denotes a curve isomorphic to $\mathbb{P}^{1}$ of specified self-intersection number; the solid ones are the components of $D$, the dashed ones are $A_{1}$ and $A_{2}$; and $\left[(2)_{m}\right]$ is short for a chain of $m(-2)$-curves (see Section $2 \mathrm{~A}$ ).

If $p_{1}, p_{2} \neq 1$ or $p_{1}=p_{2}=1$, then $(X, D)$ is a standard completion of $S_{p_{1}, p_{2}}$ in the sense of [FKZ07], see Section 2D. To obtain a standard completion in the remaining case, blow up at the preimage of the point $(\infty, \infty)$ and contract $L_{1, \infty}+L_{2,0}$ : this gives a graph as in Figure 1(b). In Corollary 2.6(b) we use [FKZ07, Corollary 3.36] to prove that $S_{p_{1}, p_{2}} \neq S_{p_{1}^{\prime}, p_{2}^{\prime}}$ for $\left\{p_{1}, p_{2}\right\} \neq\left\{p_{1}^{\prime}, p_{2}^{\prime}\right\}$ just by comparing these graphs.

In Section 4 we describe the diffeomorphism types of surfaces in $\mathcal{S}_{0}$. The result is summarized in Theorem 1.4. We refer to $[\mathrm{GS} 99, \S 4,5]$ for a language of Kirby diagrams (see also Section 4A) and use the notation of [BW01] for 2-bridge knots.

Theorem 1.4. The surface $S_{p_{1}, p_{2}}$ from Theorem 1.1 is diffeomorphic to the interior of a 2-handlebody on a 0 -framed 2-bridge knot $K_{\left[2 d_{1}, 2 d_{2}\right]}$, where $d_{j}=\operatorname{deg} p_{j}+1, j \in\{1,2\}$. Moreover, $S_{p_{1}, p_{2}}$ is not homeomorphic to $S_{p_{1}^{\prime}, p_{2}^{\prime}}$ for $\left\{\operatorname{deg} p_{1}, \operatorname{deg} p_{2}\right\} \neq\left\{\operatorname{deg} p_{1}^{\prime}, \operatorname{deg} p_{2}^{\prime}\right\}$.

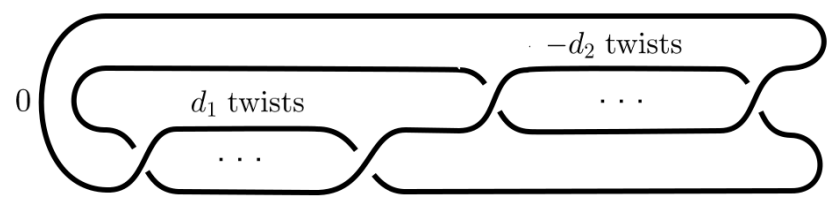

FiguRE 2. Kirby diagram of a 4-manifold whose interior is $S_{p_{1}, p_{2}}$ with $\operatorname{deg} p_{j}=d_{j}-1$.

Theorem 1.4 follows by some elementary handle slides from Proposition 4.3, where we translate Construction 1.3 to the Kirby diagram in Figure 3.

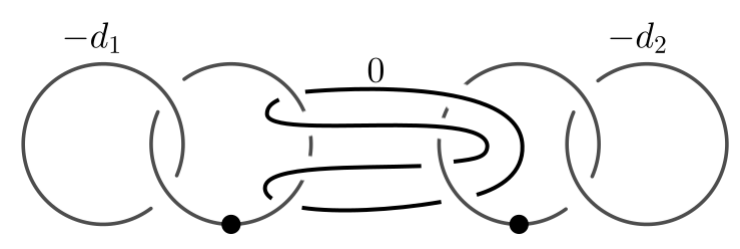

Figure 3. Kirby diagram equivalent to the one in Figure 2, cf. [GS99, Fig. 5.14].

In Corollary 2.10 we prove that $S_{p_{1}, p_{2}}$ for different degrees of $p_{j}$ are not homeomorphic, because they have different homotopy types at infinity, see [Fuj82, 1.21]. Like in the proof of Theorem 1.1, we deduce it from the graph of $D$, this time applying the calculus of graph 3-manifolds [Neu81] to $M=\partial \operatorname{Tub}(D)$, see [Mum61]. That argument becomes more explicit once it is related to the fact that, by Theorem 1.4, $M$ is a 0 -surgery on $K_{\left[2 d_{1}, 2 d_{2}\right]}$. We exploit it in Section $4 \mathrm{C}$, giving a thorough geometric description of $M$. The fundamental group $\pi_{1}(M)=\pi_{1}^{\infty}\left(S_{p_{1}, p_{2}}\right)$ is computed in Proposition 4.5.

Remark 1.5. We do not know which of the $S_{p_{1}, p_{2}}$, for fixed degrees of $p_{1}, p_{2}$, are biholomorphic.

An interesting homogeneity property of $S_{1,1}$ was shown in [KK11, §8]: although $S_{1,1}$ has no algebraic automorphisms except $\left(x_{1}, x_{2}\right) \mapsto\left(x_{2}, x_{1}\right)$, it admits a lot of nice holomorphic ones, coming from the flows of algebraic vector fields. Theorem 1.6 shows that the same holds for all $S_{p_{1}, p_{2}}$. Denote by AAut ${ }_{\text {hol }}\left(S_{p_{1}, p_{2}}\right)$ the subgroup of $\operatorname{Aut}_{\text {hol }}\left(S_{p_{1}, p_{2}}\right)$ generated by elements of flows of complete algebraic vector fields on $S_{p_{1}, p_{2}}$. 
Theorem 1.6. We keep notation from Construction 1.3.

(a) If $p_{1} \neq p_{2}$ then $\operatorname{Aut}\left(S_{p_{1}, p_{2}}\right)$ is trivial. Otherwise, $\operatorname{Aut}\left(S_{p, p}\right)=\mathbb{Z}_{2}$ is generated by $\left(x_{1}, x_{2}\right) \mapsto\left(x_{2}, x_{1}\right)$.

(b) The group AAuthol $\left(S_{p_{1}, p_{2}}\right)$ acts m-transitively, for any $m$, on its open orbit $S_{p_{1}, p_{2}} \backslash Z$, where:

(i) $Z=\emptyset$ if $p_{1}=p_{2}=1$,

(ii) $Z=\left(\left(L_{3-j, 1} \cap A_{j}\right) \cup A_{3-j}\right) \cap S_{p_{1}, p_{2}} \cong\{\mathrm{pt}\} \sqcup \mathbb{C}^{1}$ if $p_{j}=1, p_{3-j} \neq 1$ for some $j \in\{1,2\}$,

(iii) $Z=\left(A_{1} \cup A_{2}\right) \cap S_{p_{1}, p_{2}} \cong \mathbb{C}^{1} \sqcup \mathbb{C}^{1}$ if $p_{1}, p_{2} \neq 1$.

Part (a) is obtained as Corollary 2.6(c) from the uniqueness of standard boundary. To prove (b) it suffices, by [KKL20, Theorem 1.6], to describe all $\mathbb{C}^{*}$-fibrations of $S_{p_{1}, p_{2}}$ : this is done in Proposition 5.5.

We now explain what is missing in the proof of [GM88, Theorem 2], and how we are going to correct it. The aforementioned result is deduced from the (partial, but sufficient) classification of almost minimal pairs of Kodaira dimension zero given in [Fuj82, §8]. For the general statement see [Koj99].

More precisely, let $S \in \mathcal{S}_{0}$ and let $\left(X_{0}, D_{0}\right)$ be a smooth completion of $S$. Then by the Miyanishi's theory of peeling (see [Miy01, p. 107] or Section 2F below), there is a sequence of birational morphisms

$$
\left(X_{0}, D_{0}\right) \stackrel{\psi_{1}}{\longrightarrow}\left(X_{1}, D_{1}\right) \stackrel{\psi_{2}}{\longrightarrow} \ldots \stackrel{\psi_{n}}{\longrightarrow}\left(X_{n}, D_{n}\right),
$$

such that $\left(X_{n}, D_{n}\right)$ is almost minimal, hence known, and each $\psi_{i}$ is an snc-minimalization of $D_{i-1}+A$, where $A \subseteq X_{i-1}$ is a (-1)-curve not contained in $D_{i-1}$ such that $A \cdot D_{i-1}=1$. The latter process is called a half-point attachment in [Fuj82, 8.15]. Therefore, to find $S$ it suffices to identify an $\left(X_{n}, D_{n}\right)$ on the list in [Fuj82, 8.70] (cf. [Koj99, Proposition 1.5]), and perform suitable half-point attachments.

The proof in [GM88, Theorem 2] proceeds exactly along these lines. However, what is meant in loc. cit. by a half-point attachment is just one blowup, the contraction of $A$ (cf. e.g. [FZ94, Definition 4.15]). Nonetheless, if $A$ meets a (-2)-curve $C \subseteq D_{i-1}$ which is not branching in $D_{i-1}$, then after the contraction of $A$, the image of $D_{i-1}$ is not snc-minimal, and $\psi_{i}$ should contract the image of $C$, too.

This is why in [GM88] only $S_{1,1}$ is reconstructed. Indeed, it is shown (essentially) that $n=2, X_{2}=$ $\mathbb{P}^{1} \times \mathbb{P}^{1}$ and $D_{2}$ is a union of two vertical and two horizontal lines. But $(X, D) \longrightarrow\left(X_{2}, D_{2}\right)$ is obtained by two blowups at smooth points of $D_{2}$, see Figure $1(\mathrm{c})$. The smooth completions of $S_{p_{1}, p_{2}}$, which are obtained from $\left(X_{2}, D_{2}\right)$ by two sequences of blowups over $D_{2}$, see Figure 1 (a), are missing.

In Section 3 we repeat the above proof, filling in the missing part. Our approach contains no essentially new ingredient. However, since the assumption $S \in \mathcal{S}_{0}$ is substantially stronger than just $\kappa(S)=0$, we do not need to rely on the structure theorem [Fuj82, 8.70], as it is done in [GM88]. So for the convenience of the reader, we make our proof more self-contained. The only general result we refer to is the theorem of Kawamata [Kaw79, 2.2] which asserts that if $\kappa(S)=0$ then the positive part of the Zariski decomposition of $K_{X_{n}}+D_{n}$ is numerically trivial, see [Miy01, II.6.2.1].

Acknowledgments. We would like to thank the referee for valuable comments, including pointing out a flaw in the earlier version of the proof. The first author would like to thank prof. Frank Kutzschebauch for drawing his attention to the article [FKN19] and for many insightful discussions on the subject.

\section{Preliminaries}

\section{A. Log surfaces}

We now briefly recall the language of log surfaces. For a complete introduction we refer to [Fuj82].

By a curve we mean an irreducible, reduced variety of dimension 1.

Let $X$ be a smooth projective surface. A curve $C \subseteq X$ is called an $n$-curve if $C \cong \mathbb{P}^{1}$ and $C^{2}=n$. In particular, a (-1)-curve is an exceptional curve of a blowup, and a 0 -curve is a fiber of a $\mathbb{P}^{1}$-fibration.

Let $D$ be an effective $\mathbb{Q}$-divisor on $X$. By a component of $D$ we always mean an irreducible component. The number of components of $D$ is denoted by $\# D$. The branching number of a component $C \subseteq D$ is

$$
\beta_{D}(C)=C \cdot(D-C) .
$$

We say that $C$ is a tip of $D$ if $\beta_{D_{\text {red }}}(C)=1$, and branching in $D$ if $\beta_{D_{\text {red }}}(C) \geqslant 3$.

Assume that $D$ is simple normal crossing (snc), that is, $D$ is reduced, all components of $D$ are smooth and meet transversally, at most two at each point. A $(-1)$-curve $C \subseteq D$ is called superfluous if $1 \leqslant$ $\beta_{D}(C) \leqslant 2$ and $C$ meets each component of $D$ at most once: in other words, after contraction of $C$, the image of $D$ remains snc. We say that $D$ is snc-minimal if it contains no superfluous $(-1)$-curves. An snc-minimalization of $D$ is the contraction of all superfluous $(-1)$-curves in $D$ and its images.

Assume further that $D$ has connected support and $\beta_{D}(C) \leqslant 2$ for all components $C$ of $D$. We say that $D$ is a chain if at least one inequality is strict, otherwise we say that $D$ is circular. We order the 
components $T_{1}, \ldots, T_{m}$ of $D$ in such a way that $T_{i} \cdot T_{i+1}=1$ for $i \in\{1, \ldots, m-1\}$, and $T_{1}$ is a tip of $D$ in case $D$ is a chain. The sequence of integers $\left(-T_{1}^{2}, \ldots,-T_{m}^{2}\right)$ is then called a type of $D$. We often abuse notation and write

$$
D=\left[-T_{1}^{2}, \ldots,-T_{m}^{2}\right] \text { if } D \text { is a chain and } D=\left(\left(-T_{1}^{2}, \ldots,-T_{m}^{2}\right)\right) \text { if } D \text { is circular. }
$$

A sequence consisting of an integer $a$ repeated $k$ times will be abbreviated as $(a)_{k}$.

An effective divisor $D$ is called a tree if $D_{\text {red }}$ is snc and has no circular subdivisor. A fork is a tree with exactly one branching component and three maximal twigs. A rational tree (chain, fork...) is a tree (chain, fork...) whose components are all rational.

We say that a reduced divisor $D=\sum D_{i}$ is negative definite if its intersection matrix $\left[D_{i} \cdot D_{j}\right]_{1 \leqslant i, j \leqslant r}$ is.

Let again $D$ be an snc divisor. A chain $T \subseteq D$ is a twig of $D$ if it contains a tip of $D$ and its components are non-branching in $D$. We order a twig in such a way that its first component is a tip of $D$. A twig is called admissible if all its components are rational and have self-intersection number at most -2 . An (admissible) twig of $D$ is maximal if it is maximal in the set of (admissible) twigs ordered by inclusion of supports. A (-2)-twig is a twig whose components are (-2)-curves.

Let $T$ be an admissible twig of $D$. Then $T$ is negative definite, so there is a unique $\mathbb{Q}$-divisor $\mathrm{Bk}_{D}(T) \leqslant$ $T$, called the bark of $T$, such that for every component $T_{0}$ of $T$

$$
T_{0} \cdot \mathrm{Bk}_{D}(T)=T_{0} \cdot\left(K_{X}+D\right),
$$

that is, $T_{0} \cdot \mathrm{Bk}_{D}(T)=-1$ if $T_{0}$ is a tip of $D$ and $T_{0} \cdot \mathrm{Bk}_{D}(T)=0$ otherwise. An important result for the theory of peeling asserts that the coefficients of $\mathrm{Bk}_{D}(T)$ are strictly between 0 and 1 [Miy01, II.3.3].

Assume that $D$ is a connected snc-minimal divisor which is not negative definite. Then $\operatorname{Bk} D$ is defined as the sum of barks of all maximal admissible twigs of $D$. We put $D^{\#}=D-\operatorname{Bk} D$.

Let $D$ be an snc divisor on $X$. We say that a blowup $X^{\prime} \longrightarrow X$ at a point $p \in D$ is outer with respect to $D$ if $p$ is a smooth point of $D$, otherwise it is called inner. We say that a birational map $X \rightarrow Y$ touches a divisor if it is not an isomorphism in any of its neighborhoods.

A log smooth pair $(X, D)$ consists of a smooth projective surface $X$ and an snc divisor $D$ on $X$. Any smooth surface $S$ admits a $\log$ smooth completion, that is, a $\log$ smooth pair $(X, D)$ such that $S \cong X \backslash D$. We say that such a completion is minimal if it does not dominate birationally any other $\log$ smooth completion, or, equivalently, if $D$ is snc-minimal.

\section{B. Affine surfaces whose coordinate rings are factorial and have trivial units}

For a divisor $D$ on a smooth surface $X$, we denote by $\mathbb{Z}[D]$ the free abelian group whose basis is the set of components of $D$. There is a natural group homomorphism $\mathbb{Z}[D] \longrightarrow \operatorname{Pic}(X)$. The following lemma is well known, cf. [Fuj82, 1.17] or [GM88, Lemma 2.2].

Lemma 2.1 (Criterion for $\left.S \in \mathcal{S}_{\kappa}\right)$. Let $(X, D)$ be a smooth completion of a smooth affine surface $S$.

(a) $\mathbb{C}[S]$ is a UFD if and only if $\mathbb{Z}[D] \longrightarrow \operatorname{Pic}(X)$ is surjective.

(b) $\mathbb{C}[S]^{*}=\mathbb{C}^{*}$ if and only if $\mathbb{Z}[D] \longrightarrow \operatorname{Pic}(X)$ is injective.

Assume that $S \in \mathcal{S}_{\kappa}$. Then $\mathbb{Z}[D]=\operatorname{Pic}(X)=\operatorname{NS}(X)$ and $q(X)=0$. If $\kappa<2$ then $X$ is rational.

Proof. (a) By [Har77, II.6.2], $\mathbb{C}[S]$ is factorial if and only if the divisor class group $\mathrm{Cl}(S)$ of $S$ is trivial. It follows from [Har77, II.6.5(c)] that $\mathrm{Cl}(S)$ is the cokernel of the natural map $\mathbb{Z}[D] \longrightarrow \mathrm{Cl}(X)$. Eventually, $\mathrm{Cl}(X)=\operatorname{Pic}(X)$ because $X$ is smooth [Har77, II.6.16].

(b) A regular function on $S$ is invertible if and only if it extends to $f \in \mathbb{C}(X)$ with all zeros and poles in $D$, that is, with $\operatorname{div}(f) \in \mathbb{Z}[D]$. Two rational functions on $X$ with the same divisor differ by an element of $\mathcal{O}_{X}(X)^{*}=\mathbb{C}^{*}$, so $f \mapsto \operatorname{div}(f)$ gives an isomorphism of $\mathbb{C}[S]^{*} / \mathbb{C}^{*}$ with the kernel of $\mathbb{Z}[D] \longrightarrow \operatorname{Pic}(X)$.

For the remaining part, we follow [GM88, Lemma 2.2]. Assume that $S \in \mathcal{S}_{\kappa}$. Then (a), (b) give $\mathbb{Z}[D]=\operatorname{Pic}(X)$. The exponential sequence induces an exact sequence

$$
0 \longrightarrow H^{1}(X, \mathbb{Z}) \longrightarrow H^{1}\left(X, \mathcal{O}_{X}\right) \longrightarrow \operatorname{Pic}(X) \longrightarrow H^{2}(X, \mathbb{Z})
$$

Since $H^{1}\left(X, \mathcal{O}_{X}\right)$ is a vector space over $\mathbb{C}$, and by (a) $\operatorname{Pic}(X)$ is a finitely generated abelian group, we obtain $q(X)=h^{1}\left(X, \mathcal{O}_{X}\right)=0$ and that $\operatorname{Pic}(X) \longrightarrow H^{2}(X, \mathbb{Z})$ is injective, i.e. $\operatorname{Pic}(X)=\operatorname{NS}(X)$. Now to prove that $X$ is rational, it remains to show that $p_{g}(X)=0$ [Har77, IV.6.2]. Assume $\kappa<2$. Because $S$ is affine, $D$ supports an effective ample divisor, so if $\left|K_{X}\right| \neq \emptyset$ then for $m \gg 0$ we have $h^{0}\left(m\left(K_{X}+D\right)\right) \sim m^{2}$, hence $\kappa=2$; a contradiction. 
Remark 2.2. Lemma 2.1(a) implies that an open affine subset of a smooth factorial surface is factorial, and that the converse holds if and only if its complement is a principal divisor. This is a special case of the Nagata lemma, see e.g. [Eis95, Lemma 19.20]

Corollary $2.3\left(S_{p_{1}, p_{2}} \in \mathcal{S}_{\kappa}\right)$. Let $(X, D)$ be the log smooth completion of $S_{p_{1}, p_{2}}$ as in Construction 1.3. Then $\operatorname{Pic}(X)=\mathbb{Z}[D]$. In particular, $\mathbb{C}\left[S_{p_{1}, p_{2}}\right]$ is a UFD and $\mathbb{C}\left[S_{p_{1}, p_{2}}\right]=\mathbb{C}^{*}$.

Proof. We use the notation introduced in Construction 1.3. The group $\operatorname{Pic}(X)$ is generated by the components of $\varphi^{*}\left(\ell_{1, \infty}+\ell_{1,0}+\ell_{2, \infty}+\ell_{2,0}\right)_{\text {red }}=D+A_{1}+A_{2}$, with relations $0=\varphi^{*}\left(\ell_{j, 0}-\ell_{j, \infty}\right)=$ $A_{j}+T_{j}+L_{j, 0}-L_{j, \infty}, j \in\{1,2\}$, where $T_{j}$ is the twig of $D$ meeting $L_{j, 0}$. Therefore, $\operatorname{Pic}(X)=\mathbb{Z}[D+$ $\left.A_{1}+A_{2}\right] / \mathbb{Z}\left[A_{1}+A_{2}\right]=\mathbb{Z}[D]$. The remaining assertion follows from Lemma 2.1.

\section{C. $\mathbb{P}^{1}$-fibrations}

A fibration of a smooth surface $S$ is a surjective morphism onto a curve whose general fiber is irreducible and reduced. A curve on $S$ is called vertical (resp. horizontal) if $C \cdot F=0$ (resp. $C \cdot F>0$ ) for a fiber $F$; it is called an $n$-section if $C \cdot F=n$. Any divisor $D$ decomposes uniquely as $D=D_{\text {vert }}+D_{\text {hor }}$, where all components of $D_{\text {vert }}$ are vertical and all components of $D_{\text {hor }}$ are horizontal.

A $\mathbb{P}^{1}$ - (resp. $\mathbb{C}^{1}-, \mathbb{C}^{*}$ ) fibration is a fibration whose general fiber is isomorphic to $\mathbb{P}^{1}$ (resp. $\mathbb{C}^{1}, \mathbb{C}^{*}:=$ $\left.\mathbb{C}^{1} \backslash\{0\}\right)$. A fiber not isomorphic to a general one will be called degenerate.

The surfaces in $\mathcal{S}_{0}$ do not admit $\mathbb{C}^{1}$-fibrations by the Iitaka Easy Addition Theorem [Iit82, Theorem 11.9], but they admit plenty of $\mathbb{C}^{*}$-fibrations (eg. produced by Lemma 3.1(a)), which we will use in Proposition 5.5(c) as first integrals for complete algebraic vector fields, see [KKL20, Theorem 1.6]. The structure of $\mathbb{C}^{*}$-fibrations is well described in [Fuj82, §7], however, for our purposes it will be more convenient to study them directly, by completing them to $\mathbb{P}^{1}$-fibrations.

Let $F$ be a degenerate fiber of a $\mathbb{P}^{1}$-fibration of a smooth projective surface. Then $F$ is obtained from a 0-curve by a sequence of blowups, hence its geometry is easy to understand, see eg. [Fuj82, §4]. In particular, $F$ is a rational tree with no branching $(-1)$-curves; moreover, if a (-1)-curve has multiplicity 1 in $F$ then it is a tip of $F$, and $F$ contains another $(-1)$-curve [Fuj82, 7.3].

The following observation, see [Fuj82, 7.14(1)], will be used several times.

Lemma 2.4 (our $\mathbb{C}^{*}$-fibrations are untwisted). Let $(X, D)$ be a log smooth completion of a smooth affine surface $S$ such that $\mathbb{C}[S]$ is a UFD. Let $H_{1}, \ldots, H_{r}$ be all components of $D_{\text {hor }}$ for some $\mathbb{P}^{1}$-fibration of $X$. Put $h_{j}=H_{j} \cdot F$ for a fiber $F$. Then $\operatorname{gcd}\left(h_{1}, \ldots, h_{r}\right)=1$.

Proof. By Tsen's theorem, $X$ contains a 1-section $H$. By Lemma 2.1(a), $H \equiv V+\sum_{j=1}^{r} a_{j} H_{j}$ for some vertical $V$ and $a_{1}, \ldots, a_{r} \in \mathbb{Z}$. Intersecting with $F$ gives $1=\sum_{j=1}^{r} a_{j} h_{j}, \operatorname{sog} \operatorname{gcd}\left(h_{1}, \ldots, h_{r}\right)=1$.

\section{D. Standard completions and elementary transformations}

A minimal log smooth completion of an open surface may not be unique. However, [FKZ07, Definition 2.13] distinguishes a class of standard completions, which gives a convenient tool to tell affine surfaces apart. We now recall this definition. A rational chain is standard if it is of type

$$
\left[(0)_{2 k+1}\right] \text { or }\left[(0)_{2 k}, a_{1}, \ldots, a_{l}\right] \text { for some integers } k, l \geqslant 0 \text { and } a_{1}, \ldots, a_{l} \geqslant 2 \text {. }
$$

A rational circular divisor is standard if it is of type

$$
\left(\left((0)_{2 k}, a_{1}, \ldots, a_{l}\right)\right) \text { or }\left(\left((0)_{k}, a\right)\right) \text { or }\left(\left((0)_{2 k}, 1,1\right)\right) \text { for some } k, l \geqslant 0, a \geqslant 0 \text { and } a, a_{1}, \ldots, a_{l} \geqslant 2 \text {. }
$$

Let now $D$ be any snc divisor, and let $B$ be the sum of all components of $D$ which are either branching or non-rational; so every connected component of $D-B$ is rational, chain or circular. We say that $D$ is standard if every connected component of $D-B$ is standard in the above sense. A log smooth completion $(X, D)$ is standard if $D$ is. Note that the $\log$ smooth completions of $S_{p_{1}, p_{2}}$ in Figure 1 are standard.

Let $D$ be an snc divisor on $X$, and let $C \subseteq D$ be a non-branching 0-curve. If $\beta_{D}(C)=2$ choose $p \in C \cap(D-C)$, otherwise let $p$ be any point of $C$. A flow (or elementary transformation) on $C$ is the birational map $(X, D) \rightarrow\left(X^{\prime}, D^{\prime}\right)$, where $D^{\prime}$ is the reduced total transform of $D$, defined as a blowup at $p$, followed by the contraction of the proper transform of $C$. We remark that in Section 5 we will use the word "flow" in its standard meaning, i.e. flow of a vector field: this will lead to no confusion.

Let $\varphi:(X, D) \rightarrow\left(X^{\prime}, D^{\prime}\right)$ be a flow on $C \subseteq D$. Then $\left.\varphi\right|_{X \backslash D}: X \backslash D \longrightarrow X^{\prime} \backslash D^{\prime}$ is an isomorphism. In particular, if the components of $D$ generate $\operatorname{Pic}(X)$ (respectively, are $\mathbb{Z}$-linearly independent in $\operatorname{Pic}(X)$ ), then by Lemma 2.1 the same is true for the components of $D^{\prime}$ in $\operatorname{Pic}\left(X^{\prime}\right)$. Moreover, $\varphi$ does not touch components of $D$ other than $C$ and the components of $D$ meeting $C$. If $C$ is not a tip of $D$, then $\varphi$ replaces a subchain $[a, 0, b] \subseteq D$, whose middle component is $C$, by $[a+1,0, b-1] \subseteq D^{\prime}$. 
We are ready to formulate the result used to distinguish the surfaces $S_{p_{1}, p_{2}}$ for different $\left\{p_{1}, p_{2}\right\}$.

Lemma 2.5 ([FKZ07, Corollary 3.36]). Any smooth affine surface admits a standard log smooth completion. Any two such completions differ by a sequence of flows on some 0-curves in the boundary.

Corollary $2.6\left(S_{p_{1}, p_{2}}\right.$ are non-isomorphic). Let $S_{p_{1}, p_{2}}$ be as in Theorem 1.1. Then

(a) The standard completion $(X, D)$ of $S_{p_{1}, p_{2}}$ is unique up to an isomorphism, with $D$ as in Figure 1.

(b) The surfaces $S_{p_{1}, p_{2}}$ and $S_{p_{1}^{\prime}, p_{2}^{\prime}}$ are not isomorphic unless $\left\{p_{1}, p_{2}\right\}=\left\{p_{1}^{\prime}, p_{2}^{\prime}\right\}$.

(c) If $p_{1} \neq p_{2}$ then $\operatorname{Aut}\left(S_{p_{1}, p_{2}}\right)$ is trivial. Otherwise, $\operatorname{Aut}\left(S_{p, p}\right)=\mathbb{Z}_{2}$ is generated by $\left(x_{1}, x_{2}\right) \mapsto\left(x_{2}, x_{1}\right)$.

Proof. (a) By Lemma 2.5, D differs from the one in Figure 1 by a sequence of flows. Because all twigs of the one in Figure 1 are admissible, they are not touched by any flow. Since a flow preserves the non-weighted graph and the sum of self-intersection numbers; we only need to check that the latter agree.

Consider the case when $p_{1}, p_{2} \neq 1$ or $p_{1}=p_{2}=1$. Then any such $D$ contains a rational circular divisor $L$ with components, say, $L_{1}, L_{2}, L_{3}, L_{4}$, such that $L_{i} \cdot L_{i+1}=1$ for $i \in\{1,2,3\}$; if $p_{j} \neq 1$ for $j \in\{1,2\}$ then $L_{2+j}$ meets a twig of $D$ of type $\left[(2)_{\operatorname{deg} p_{j}}\right]$, and $L_{1}^{2}=0$, i.e. $L_{1}$ is the 0 -curve on which the last flow is performed. If $p_{1}, p_{2} \neq 1$ then the chain $L_{1}+L_{2}$ is standard, and if $p_{1}=p_{2}=1$ then the whole $L$ is standard. In any case, we infer that $L_{2}^{2}=0$. It follows that $L_{3}^{2}, L_{4}^{2}<0$ : indeed, for $i \in\{1,2\}, L_{i+2}$ is vertical for the $\mathbb{P}^{1}$-fibration of $X$ induced by $\left|L_{i}\right|$, and it is not a fiber because otherwise $L_{i+2}=L_{i}$ in $\operatorname{Pic}(X)$, contrary to Lemma 2.1(b). Because the sum $L_{1}^{2}+L_{2}^{2}+L_{3}^{2}+L_{4}^{2}$ is not changed by a flow, we get $L_{3}^{2}+L_{4}^{2}=-2$, so $L_{3}^{2}=L_{4}^{2}=-1$, as claimed.

In case $p_{j} \neq 1, p_{3-j}=1$ we similarly obtain that $D$ has a rational circular divisor $L_{1}+L_{2}+L_{3}$, where $L_{3}$ meets a twig $\left[(2)_{\operatorname{deg} p_{j}}\right]$ and $L_{1}$ is a 0 -curve. The chain $L_{1}+L_{2}$ is standard, so $L_{2}^{2}=0$, and $L_{3}^{2}=L_{1}^{2}+L_{2}^{2}+L_{3}^{2}=1$.

(b), (c) Let $S_{p_{1}, p_{2}} \longrightarrow S_{p_{1}^{\prime}, p_{2}^{\prime}}$ be an isomorphism. By Lemma 2.5, it extends to an isomorphism, say $\tau:\left(X_{1}, D_{1}\right) \longrightarrow\left(X_{2}, D_{2}\right)$, between their standard completions, which by (a) are both as in Construction 1.3. If they are as in Figure 1(b), we reverse the last step of Construction 1.3 and lift $\tau$ accordingly, so that $\left(X_{i}, D_{i}\right)$ are as in Figure 1(a) or 1(c) (but possibly no longer standard).

For $i, j \in\{1,2\}$ let $A_{j, i} \subseteq X_{i}$ be as in Figure 1. Because by Lemma 2.1 $\operatorname{Pic}\left(X_{i}\right)=\mathbb{Z}\left[D_{i}\right]$, the curve $\tau\left(A_{j, 1}\right)$ is linearly equivalent to $A_{j, 2}$. In fact, $\tau\left(A_{j, 1}\right)=A_{j, 2}$ because $\tau\left(A_{j, 1}\right) \cdot A_{j, 2}=A_{j, 2}^{2}<0$. Therefore, $\tau$ descends to an automorphism $\tau_{0}$ of $\mathbb{P}^{1} \times \mathbb{P}^{1}$ fixing $\ell_{1,0}+\ell_{1, \infty}+\ell_{2,0}+\ell_{2, \infty}$ and the points $(0,1),(1,0)$, so $\tau_{0}\left(x_{1}, x_{2}\right)=\left(x_{1}, x_{2}\right)$ or $\left(x_{2}, x_{1}\right)$. Hence $\tau$ or $\tau \circ \varepsilon$, where $\varepsilon\left(x_{1}, x_{2}\right)=\left(x_{2}, x_{1}\right)$, is trivial on each exceptional curve of $\varphi: X \longrightarrow \mathbb{P}^{1} \times \mathbb{P}^{1}$. This shows that $\left\{p_{1}, p_{2}\right\}=\left\{p_{1}^{\prime}, p_{2}^{\prime}\right\}$ and $\tau=$ id or $\varepsilon$.

Remark $2.7\left(S_{p_{1}, p_{2}}\right.$ are stably non-isomorphic, cf. [FKN19, Corollary 4.3]). Because $\kappa\left(S_{p_{1}, p_{2}}\right)=0$ by Corollary 2.13 below, [IF77] implies that $S_{p_{1}, p_{2}} \times \mathbb{C}^{n} \neq S_{p_{1}^{\prime}, p_{2}^{\prime}} \times \mathbb{C}^{n}$ for any $n \geqslant 0$ and $\left\{p_{1}, p_{2}\right\} \neq\left\{p_{1}^{\prime}, p_{2}^{\prime}\right\}$.

We conclude with a well-known application of flows, which will be useful in the proof of Lemma 3.1.

Lemma 2.8 ([Fuj82, 6.13], cf. [FZ94, Lemma 4.14]). Let $(X, D)$ be a $\log$ smooth pair with $\kappa(X \backslash D) \geqslant 0$. Let $T$ be a rational twig of $D$. Then $C^{2} \leqslant-1$ for every component $C$ of $T$. In particular, if $D$ is snc-minimal then all rational twigs of $D$ are admissible.

Proof. Let $T_{k}$ be the $k$-th component of $T$, put $T_{0}=0$. Suppose $T_{k}^{2} \geqslant 0$ for some $k \in\{1, \ldots, \# T\}$. Write $\{p\}=T_{k} \cap\left(D-T_{k}-T_{k-1}\right)$, or choose $p \in T_{k} \backslash T_{k-1}$ if that intersection is empty. Blowing up $T_{k}^{2}$ times at $p$ and its infinitely near points on the proper transforms of $T_{k}$, we can assume that $T_{k}=[0]$. If $k>1$, then after $\pm T_{k-1}^{2}$ flows on $T_{k}$, we can assume $T_{k-1}=[0]$, too. Thus by induction, we can assume $k=1$, i.e. $T_{1}=[0]$ is a tip of $D$. Then $\left|T_{1}\right|$ induces a $\mathbb{P}^{1}$-fibration of $X$ which restricts to a $\mathbb{C}^{1}$-fibration of $X \backslash D$, so $\kappa(X \backslash D)=-\infty$ by the Iitaka Easy Addition theorem; a contradiction.

\section{E. Graph 3-manifolds and their normal forms}

An important topological invariant of an affine surface $S$ is its fundamental group at infinity, $\pi_{1}^{\infty}(S):=$ $\lim _{1} \pi_{1}(S \backslash K)$, where $\varliminf_{\text {im }}$ runs over all compact $K \subseteq S$. If $(X, D)$ is a log smooth completion of $S$, then $\overleftarrow{\pi_{1}^{\infty}}(S)=\pi_{1}(M)$, where $M$ is the boundary of a nice tubular neighborhood of $D$, constructed in [Mum61] as a plumbed manifold whose graph is the dual graph of $D$, see [GS99, 4.6.2]. More precisely, $\operatorname{Tub}(D)$ is a union, taken over components $C$ of $D$, of disk bundles $\xi_{C}$ with Euler numbers $C^{2}$, where for any component $C^{\prime}$ of $D$ meeting $C$, a fiber of $\xi_{C}$ over $C \cap C^{\prime}$ is glued to a neighborhood of $C \cap C^{\prime}$ in $C$.

For a general introduction to 3-manifolds we refer to [Hat07, AFW15]. We denote by $\mathbb{S}^{k}, \mathbb{D}^{k}$ the (real) $k$-dimensional sphere and disk, respectively, and by $\mathbb{T}^{k}=\left(\mathbb{S}^{1}\right)^{k}$ the $k$-dimensional torus. We say that a 3 -manifold is prime if it cannot be written as a connected sum of two 3-manifolds other than $\mathbb{S}^{3}$, and irreducible if any embedded $\mathbb{S}^{2}$ bounds a ball. A prime, oriented 3-manifold is either irreducible or $\mathbb{S}^{1} \times \mathbb{S}^{2}$. 
If $M$ is prime, and not a lens space (which will be the case for $S=S_{p_{1}, p_{2}}$ ), then $\pi_{1}(M)$ determines $M$ uniquely, up to a diffeomorphism [AFW15, Theorem 2.1.2]. Therefore, in this case to distinguish the homeomorphism type of $S$ at infinity, it suffices to describe $\pi_{1}(M)$, which, in turn, is given by the graph of $D$, once put in a normal form [Neu81, Theorem 4.2]. The definition of normal form is similar to the one of standard boundary, but requires some more flexibility to allow more operations between 3-manifolds than just blowing up and down. We now recall the definition of graph 3-manifolds following [Neu81]. We refer to [EN85] for details and relation to the JSJ-decomposition (graph manifolds are exactly those for which the latter consists only of Seifert fibered spaces: we compute it for our $M$ in Proposition 4.7).

A graph manifold is a 3 -manifold which is a union of $\mathbb{S}^{1}$-bundles over compact surfaces, glued according to some graph, as follows. An $\mathbb{S}^{1}$-bundle over $C$ with Euler number $e$ is represented by a vertex of weight $e$ and two additional numbers $g, r$, where $g$ is the genus of $C\left(g<0\right.$ if $C$ is non-orientable) and $r=b_{0}(\partial C)$. They are skipped whenever both are zero, i.e. $C \cong \mathbb{P}^{1}$. An edge between two vertices $v_{1}, v_{2}$ represents gluing of a fiber of $v_{1}$ to a small loop around a point in a base of $v_{2}$, and vice versa. Multiple edges and loops are allowed. An edge is labeled by "+" if the gluing respects the chosen orientations, and "-" if it reverses both of them. Clearly, reversing the sign of all edges adjacent to a single vertex, or of any edge adjacent to a vertex with $g<0$, does not change the manifold. Hence we will label only the edges contained in circular subgraphs with no vertices of $g<0$, see [Neu81, p. 304].

For example, the boundary $M$ from [Mum61], described above, is a graph manifold associated to the dual graph of $D$, with all edges labeled with "+". For such graph, we use the notions of branching numbers, chains, twigs as in Section 2A, a vertex being "rational" if $g=r=0$.

A connected, rational graph $\Gamma$ is normal if the following three conditions hold. First, all non-branching vertices of $\Gamma$ have weights at most -2 . Second, if a vertex with $\beta=3$ meets two twigs of type [2] then $\Gamma$ is a fork. Third, if $\Gamma=\left(\left((2)_{k}\right)\right)$ for some $k>0$ then at least two edges are labeled with "-". To extend this definition to non-rational graphs, one needs to exclude some additional special cases, see [Neu81, §4]. We will not need this extension. In general, a graph is normal if all its connected components are.

For a graph 3-manifold $N$, [Neu81, Theorem 4.1] gives an algorithm to reduce its graph to a normal form, which we denote by $\Gamma(N)$. This algorithm uses certain operations R0-R8 defined in Proposition 2.1 loc. cit, which do not change the graph manifold. Among these, $\mathrm{R} 1$ is blowing down a vertex with weight \pm 1 , and R3 is an "absorption" of a cylinder $\left(\mathbb{D}^{1} \times \mathbb{S}^{1}\right) \times \mathbb{S}^{1}$ corresponding to a rational vertex of weight 0 and $\beta=2$. The graph $\Gamma(-N)$ can be directly computed from $\Gamma(N)$ using [Neu81, Theorem 7.1].

Now we can state the main result of [Neu81] used to distinguish $\pi_{1}^{\infty}\left(S_{p_{1}, p_{2}}\right)$ for different degrees of $p_{j}$.

Lemma 2.9 ([Neu81, Theorems 4.2, 4.3]). Let $N, N^{\prime}$ be graph 3-manifolds.

(a) $\Gamma(N)=\Gamma\left(N^{\prime}\right)$ if and only if $N$ and $N^{\prime}$ are orientation-preserving diffeomorphic.

(b) If $\Gamma$ is connected then $N$ is prime.

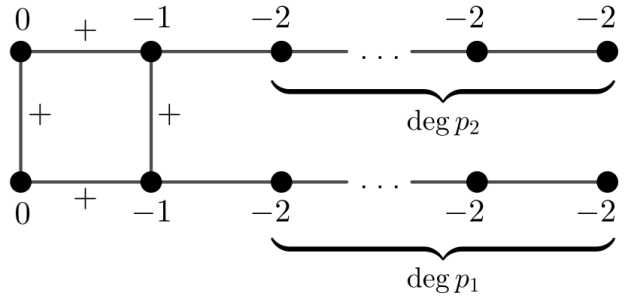

(A) Graph of $M$ as in [Mum61], see Figure 1(a)

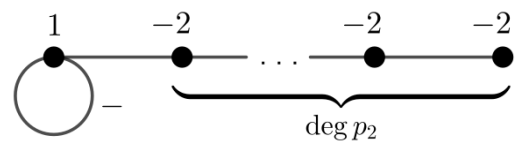

(c) $\Gamma(M)$ for $p_{1}=1, p_{2} \neq 1$

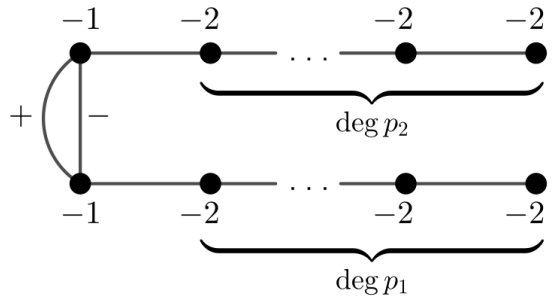

(в) $\Gamma(M)$ for $p_{1}, p_{2} \neq 1$

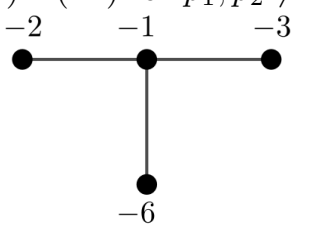

(D) $\Gamma(-M)$ for $p_{1}, p_{2}=1$, see Proposition $4.7(\mathrm{c})$

FiguRE 4. Graphs of $M=\partial \operatorname{Tub}(D)$ in the proof of Corollary 2.10

Corollary 2.10. The surfaces $S_{p_{1}, p_{2}}$ and $S_{p_{1}^{\prime}, p_{2}^{\prime}}$ are not homeomorphic for $\left\{\operatorname{deg} p_{1}, \operatorname{deg} p_{2}\right\} \neq\left\{\operatorname{deg} p_{1}^{\prime}, \operatorname{deg} p_{2}^{\prime}\right\}$.

Proof. For $S_{p_{1}, p_{2}}$ and $S_{p_{1}^{\prime}, p_{2}^{\prime}},\left\{\operatorname{deg} p_{1}, \operatorname{deg} p_{2}\right\} \neq\left\{\operatorname{deg} p_{1}^{\prime}, \operatorname{deg} p_{2}^{\prime}\right\}$ let $M, M^{\prime}$ be a boundary of tubular neighborhoods of respective $D \subseteq X$ as in Construction 1.3. Such $M$ is a graph manifold represented by Figure 4(a). Absorption (move R3 in [Neu81]) of a vertex corresponding to $L_{1, \infty}$ reduces it to a graph 
in Figure 4(b). It is in normal form if $p_{1}, p_{2} \neq 1$. If, say, $p_{1}=1$ then the normal form is obtained by blowing down (move R1) the vertex corresponding to $L_{1,0}$. If $p_{2} \neq 1$, then the resulting graph in Figure 4(c) is normal, otherwise it needs to be replaced by a standard graph of a Seifert fiber space (move R7). In our case this graph is a graph $E_{9}$, which after orientation reversing gives the one in Figure 4(d), cf. Proposition 4.7(c) and [Neu81, p. 309].

Hence $\Gamma(M) \neq \Gamma\left(M^{\prime}\right)$, so by Lemma 2.9(a) $M$ is not orientation-preserving diffeomorphic to $M^{\prime}$. Reversing orientation in Figures 4(b)-(c) inverts the edge signs and replaces a twig $\left[(2)_{d}\right]$ with $[d+2]$, so the resulting graph is different from the previous ones. Therefore, $M \neq_{\text {diffeo }} M^{\prime}$, so $M \neq_{\text {homeo }} M^{\prime}$, see [AFW15, 1.1]. Because $\Gamma(M)$ is connected and not a rational chain, $M$ is prime, and not a lens space (see [Neu81, 6.1] for lens space graphs), so by [AFW15, 2.1.2] $\pi_{1}(M) \varsubsetneqq \pi_{1}\left(M^{\prime}\right)$. The result follows because $\pi_{1}^{\infty}\left(S_{p_{1}, p_{2}}\right)=\pi_{1}(M)$ [Mum61].

\section{F. Almost minimal models}

We now recall the construction of an almost minimal model for a log smooth pair $(X, D)$. We consider only the case when $X \backslash D$ is affine, for a complete treatment we refer to [Miy01, §3], see p. 107 loc. cit. for a summary. The exposition here is based on [Pal19, §3].

The motivation for this construction to get an explicit description of a log MMP run for the pair $(X, D)$, such that the log terminal singularities on the image of $(X, D)$ are introduced as late as possible: in fact, an almost minimal model is a log resolution of the minimal model in the sense of Mori. Having understood the latter, one can use this theory to reconstruct the initial pair $(X, D)$.

The almost minimal model will be created by iterating birational morphisms of the following type.

Definition 2.11 (half-point attachment, cf. [Fuj82]). Let $(X, D)$ be a log smooth pair. Assume that

$$
A \subseteq X \text { is a }(-1) \text {-curve such that } A \nsubseteq D \text { and } A \cdot D=1 .
$$

The composition of the contraction of $A$ and new superfluous (-1)-curves in the subsequent images of $D$ :

$$
\varphi_{A}:(X, D) \longrightarrow\left(X^{\prime}, D^{\prime}\right),
$$

where $D^{\prime}=\left(\varphi_{A}\right)_{*} D$, is called a half-point attachment (of $A$ ).

Remark. In general, $\varphi_{A}$ is not uniquely determined by $A$. Indeed, it may happen that after some contraction within $\varphi_{A}$, there appear two superfluous (-1)-curves. For example, if $A$ meets the middle component $T^{\prime}$ of a subchain $T=[2,2,2]$ of $D$, whose components are non-branching in $D$, then after the contraction of $A+T^{\prime}$, both components of the image of $T$ are superfluous (-1)-curves in the image of $D$, but only one of them can be contracted.

However, such ambiguity never happens if $\mathbb{C}[X \backslash D]$ is a UFD. Indeed, Lemma 3.1(b) shows that in this case $\varphi_{A}$ contracts exactly $A$ and a maximal (-2)-twig of $D$ meeting $A$ (if such occurs).

Lemma 2.12. Let $(X, D) \longrightarrow\left(X^{\prime}, D^{\prime}\right)$ be a half-point attachment. Then for all $m \geqslant 0$ we have $h^{0}\left(m\left(K_{X^{\prime}}+D^{\prime}\right)\right)=h^{0}\left(m\left(K_{X}+D\right)\right)$. In particular, $\kappa\left(X^{\prime} \backslash D^{\prime}\right)=\kappa(X \backslash D)$.

Proof. Let $A$ be as in (2.1). We have $A \cdot\left(K_{X}+D+A\right)=-1<0$, so for any $m>0, m A$ is in the fixed locus of $\left|m\left(K_{X}+D+A\right)\right|$. Therefore, $\left|m\left(K_{X}+D\right)\right| \cong\left|m\left(K_{X}+D+A\right)\right| \cong\left|m\left(K_{X^{\prime}}+D^{\prime}\right)\right|$ because $X \longrightarrow X^{\prime}$ contracts only components of $D+A$.

Corollary 2.13. The surfaces $S_{p_{1}, p_{2}}$ from Theorem 1.1 are in $\mathcal{S}_{0}$.

Proof. By Corollary 2.3, $S_{p_{1}, p_{2}} \in \mathcal{S}_{\kappa}$ for some $\kappa$. Construction 1.3 provides a log smooth completion of $S_{p_{1}, p_{2}}$ obtained from a $\log$ smooth completion of $\mathbb{C}^{*} \times \mathbb{C}^{*}$ by two half-point attachments. Hence by Lemma $2.12, \kappa=\kappa\left(S_{p_{1}, p_{2}}\right)=\kappa\left(\mathbb{C}^{*} \times \mathbb{C}^{*}\right)=0$.

We now state the main result of the theory of peeling. Recall that $D^{\#}=D-\operatorname{Bk} D$, and $\operatorname{Bk} D$ is a $\mathbb{Q}$-effective divisor with coefficients in $(0,1)$, supported on the twigs of $D$.

Proposition 2.14 (Construction of an almost minimal model). Let $(X, D)$ be a minimal log smooth completion of a smooth affine surface. Then there is a birational morphism

$$
\psi:(X, D) \stackrel{\psi_{1}}{\longrightarrow}\left(X_{1}, D_{1}\right) \stackrel{\psi_{2}}{\longrightarrow} \ldots \stackrel{\psi_{n}}{\longrightarrow}\left(X_{n}, D_{n}\right)
$$

such that, for all $i \in\{1, \ldots, n\}$ :

(a) $\psi_{i}$ is a half-point attachment.

(b) $D_{i}=\left(\psi_{i}\right)_{*} D_{i-1}$ is snc-minimal, $\left(X_{i}, D_{i}\right)$ is $\log$ smooth and $X_{i} \backslash D_{i}$ is an affine open subset of $X \backslash D$.

(c) $h^{0}\left(m\left(K_{X_{i}}+D_{i}\right)\right)=h^{0}\left(m\left(K_{X}+D\right)\right)$ for all $m \geqslant 0$. In particular, $\kappa\left(X_{i} \backslash D_{i}\right)=\kappa(X \backslash D)$. 
(d) If $\kappa(X \backslash D) \geqslant 0$ then $K_{X_{n}}+D_{n}^{\#}$ is nef. Otherwise, either $X_{n}$ admits a $\mathbb{P}^{1}$-fibration with $\left(K_{X_{n}}+\right.$ $\left.D_{n}^{\#}\right) \cdot F<0$ for a fiber $F$; or $-\left(K_{X_{n}}+D_{n}\right)$ becomes ample after the contraction of $\mathrm{Bk} D_{n}$.

Sketch of a proof. Let $\alpha:(X, D) \longrightarrow\left(Y, D_{Y}\right)$ be the contraction of Supp Bk $D$, i.e. of all admissible twigs of $T$. Then $\alpha^{*}\left(K_{Y}+D_{Y}\right)=K_{X}+D^{\#}$. Indeed, for every component $T_{0}$ of $\mathrm{Bk} D$, we have $T_{0} \cdot\left(K_{X}+D-\alpha^{*}\left(K_{Y}+D_{Y}\right)\right)=T_{0} \cdot\left(K_{X}+T_{0}\right)+\beta_{D}\left(T_{0}\right)=-2+\beta_{D}\left(T_{0}\right)=T_{0} \cdot$ Bk $D$ by definition of bark, so $K_{X}+D-\alpha^{*}\left(K_{Y}+D_{Y}\right)=\operatorname{Bk} D$ because Bk $D$ is negative definite.

In particular, $\left(Y, D_{Y}\right)$ is $\log$ terminal. General theorems of Mori theory imply that either $\left(Y, D_{Y}\right)$ is minimal, so (d) holds for $n=0$, or there is a log extremal curve $\ell \subseteq Y$ such that $\ell^{2}<0$ and $\ell \cdot\left(K_{Y}+D_{Y}\right)<0$. Put $A=\alpha_{*}^{-1} \ell$. We claim that $A$ satisfies (2.1).

We have $0>\ell \cdot\left(K_{Y}+D_{Y}\right)=A \cdot\left(K_{X}+D^{\#}\right)$. Suppose $A \subseteq D$. Then $0>-2+\beta_{D}(A)-A \cdot \operatorname{Bk} D$, hence $A$ is a tip of $D-\operatorname{Supp~Bk~} D$ and meets at most one component of $\operatorname{Bk} D$. This means that $A$ is a component of an admissible twig of $D$, a contradiction with the definition of $\operatorname{Bk} D$.

Therefore, $A \nsubseteq D$, so $A \cdot D^{\#}>0$. Thus $A \cdot K_{X}<0$, which together with $A^{2}<0$ implies that $A$ is a $(-1)$-curve. Moreover, $A \cdot D^{\#}<-A \cdot K_{X}=1$, so $A$ meets $D$ only on Bk $D$. Now a computation [Miy01, II.3.7.1(2)], which is rather complicated, but uses nothing more than negative definiteness of $A+\operatorname{Bk} D$, shows that $A$ meets each connected component of $D$ at most once. Because $X \backslash D$ is affine, it follows that $A \cdot D=1$, so $A$ satisfies (2.1), as claimed.

We now put $\psi_{1}=\varphi_{A}:(X, D) \longrightarrow\left(X_{1}, D_{1}\right)$, for some choice of $\varphi_{A}$ such that $D_{1}$ is snc-minimal. Then for $i=1$, (a),(b) hold by definition and (c) holds by Lemma 2.12. Next, we replace $(X, D)$ by $\left(X_{1}, D_{1}\right)$ and repeat the procedure, which eventually ends since $\rho(X)$ drops after each step.

\section{Proof of Theorem 1.1}

Recall that $S_{p_{1}, p_{2}} \in \mathcal{S}_{0}$ by Corollary 2.13, and $S_{p_{1}, p_{2}} ¥ S_{p_{1}^{\prime}, p_{2}^{\prime}}$ for $\left\{p_{1}, p_{2}\right\} \neq\left\{p_{1}^{\prime}, p_{2}^{\prime}\right\}$ by Corollary 2.6(b). It remains to show that any $S \in \mathcal{S}_{0}$ is isomorphic to some $S_{p_{1}, p_{2}}$. Let $(X, D)$ be a minimal $\log$ smooth completion of $S$. The idea of the proof is to view $\varphi$ from Construction 1.3 as some almost minimalization (2.2) of $(X, D)$. By Proposition 2.14 each intermediate pair $\left(X_{i}, D_{i}\right)$ in $(2.2)$ is a log smooth completion of an affine open subset of $X \backslash D$, of the same Kodaira dimension. Hence under the assumptions of Theorem 1.1, $\kappa\left(X_{i} \backslash D_{i}\right)=0$ and $\mathbb{C}\left[X_{i} \backslash D_{i}\right]$ is a UFD by Remark 2.2.

To reconstruct $(X, D)$ we need to understand each half-point attachment $\psi_{i+1}:\left(X_{i}, D_{i}\right) \longrightarrow\left(X_{i+1}, D_{i+1}\right)$. This is done in Lemma 3.1, which is a version of [Miy01, Lemma III.4.4.3], with almost the same proof.

Lemma 3.1. Let $S$ be a smooth affine surface. Assume that $\kappa(S)=0$ and $\mathbb{C}[S]$ is UFD. Let $(X, D)$ be a minimal $\log$ smooth completion of $S$ and let $\varphi_{A}$ be a half-point attachment of some $A \subseteq X$. Then

(a) $A \cap S \cong \mathbb{C}^{1}$ is a smooth fiber of some $\mathbb{C}^{*}$-fibration of $S$

(b) $\operatorname{Exc} \varphi_{A}=\left[1,(2)_{k}\right]$, for some $k \geqslant 0$, is a twig of $D+A$. Its image does not lie on a rational twig of $\left(\varphi_{A}\right)_{*} D$.

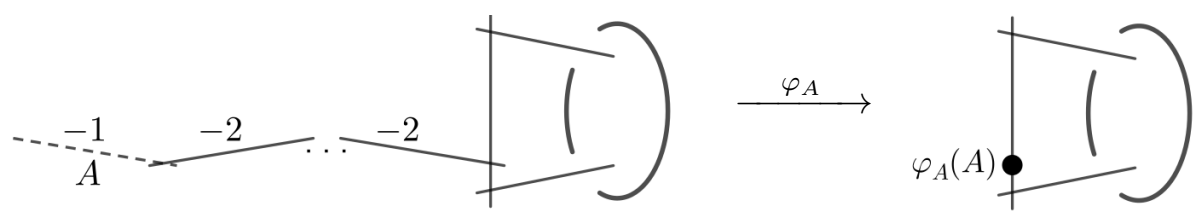

Figure 5. A half-point attachment to $S$ if $\mathbb{C}[S]$ is a UFD and $\kappa(S)=0$, see Lemma 3.1.

Proof. (a) Because $\mathbb{C}[S]$ is a UFD, $\left.A\right|_{S}$ is a divisor of zeros of some $f \in \mathbb{C}[S]$. Let $F_{\text {aff }}$ be some general fiber of $f$. It is reduced and irreducible because $\left.A\right|_{S}$ is. Put $S^{\prime}=S \backslash A, U^{\prime}=f\left(S^{\prime}\right) \subseteq \mathbb{C}^{*}$. By Lemma $2.12 \kappa\left(S^{\prime}\right)=\kappa(S)=0$, so the Kawamata addition theorem [Iit82, Theorem 11.15] gives $0=\kappa\left(S^{\prime}\right) \geqslant \kappa\left(F_{\text {aff }}\right)+\kappa\left(U^{\prime}\right) \geqslant \kappa\left(F_{\text {aff }}\right)+\kappa\left(\mathbb{C}^{*}\right)=\kappa\left(F_{\text {aff }}\right) \geqslant 0$, where the last inequality follows from the Iitaka Easy Addition theorem. Hence $\kappa\left(F_{\text {aff }}\right)=0$, which implies that $\kappa\left(F_{\text {aff }}\right) \cong \mathbb{C}^{*}$ because $F_{\text {aff }}$ is smooth and affine. Thus $f$ is a $\mathbb{C}^{*}$-fibration of $S$, with $\left.A\right|_{S}$ as one of its fibers.

(b) Let $T$ be a maximal admissible twig of $D$ meeting $A$ in its first tip; put $T=0$ if there is no such. Since $S$ is affine, $D \neq T$. Let $C$ be the component of $D-T$ meeting $A+T$. Let $\tau: \widehat{X} \longrightarrow X$ be a minimal sequence of blowups over $D$ such that the $\mathbb{C}^{*}$-fibration from (a) extends to a $\mathbb{P}^{1}$-fibration of $\widehat{X}$; let $F$ be a fiber containing $\widehat{A}:=\tau_{*}^{-1} A$. Put $\widehat{D}=\left(\tau^{*} D\right)_{\text {red. }}$. Note that $\tau$ does not touch $\widehat{A}$ : indeed, otherwise the only base point of $\tau^{-1}$ is the unique common point of $A$ and $D$, so $F \subseteq \tau^{*} A$ is negative definite, which is false. 
If a $(-1)$-curve $L \subseteq \widehat{D}_{\text {vert }}$ is superfluous in $\widehat{D}$, then $L$ is not a component of a rational twig of $\widehat{D}$. Indeed, $L \nsubseteq \mathbb{E x c} \tau$ since $\tau$ is minimal, and $\tau(L)^{2} \neq-1$ since $D$ is snc-minimal; hence $\tau(L)^{2} \geqslant 0$, and the claim follows from Lemma 2.8. Let $\sigma$ be the contraction of all vertical superfluous $(-1)$-curves in $\widehat{D}$ and its images. By our claim, $\sigma$ does not touch rational twigs of $\widehat{D}$. Put $D^{\prime}=\sigma_{*} \widehat{D}, F^{\prime}=\sigma_{*} F, A^{\prime}:=\sigma_{*} \widehat{A}$.

If $\sigma$ touches $\widehat{A}$ then $T=0$ and $\tau_{*}^{-1} C \subseteq \operatorname{Exc} \sigma$ does not lie in a rational twig of $\widehat{D}$, so the result follows. Assume $\sigma$ does not touch $\widehat{A}$, so $A^{\prime}$ is a $(-1)$-curve of multiplicity one in a fiber $F^{\prime}$. Now, $F^{\prime}-A^{\prime}$ contains another (-1)-curve, say $C^{\prime}$. By definition of $\sigma, \beta_{D^{\prime}}\left(C^{\prime}\right) \geqslant 3$. Since $\beta_{F_{\text {red }}^{\prime}}\left(C^{\prime}\right) \leqslant 2, C^{\prime}$ meets $D_{\text {hor }}^{\prime}$. By Lemma 2.4, $D_{\text {hor }}^{\prime}$ consists of two 1-sections, so $C^{\prime}$ has multiplicity one in $F^{\prime}$. Hence $\beta_{F_{\text {red }}^{\prime}}\left(C^{\prime}\right)=1$, so $C^{\prime} \cdot D_{\text {hor }}^{\prime}=2$, which implies that $\left(F^{\prime}-C^{\prime}\right) \cdot D_{\text {hor }}^{\prime}=0$, and that $C^{\prime}$ is a unique $(-1)$-curve in $F^{\prime}-A^{\prime}$. Therefore, $F^{\prime}=\left[1,(2)_{k}, 1\right]$ for some $k \geqslant 0$. In other words, $T^{\prime}:=F^{\prime}-A^{\prime}-C^{\prime}=\left[(2)_{k}\right]$ is zero or a maximal twig of $D^{\prime}$. Applying Lemma 2.8 to image of $D^{\prime}$ after contraction of $A^{\prime}+T^{\prime}$, we infer that $C^{\prime}$ does not lie in a rational twig of $D^{\prime}-T^{\prime}$.

Since $\sigma$ does not touch rational twigs of $D^{\prime}, \widehat{T}:=\sigma_{*}^{-1} T^{\prime}$ is zero or a maximal twig of $\widehat{D}$; and the component $\widehat{C}:=\sigma_{*}^{-1} C^{\prime}$ meeting it does not lie in a rational twig of $\widehat{D}-\widehat{T}$. Thus $\tau_{*} \widehat{T}=T, \tau_{*} \widehat{C}=C$ : indeed, otherwise $\tau$ touches $\widehat{C}$, so $C^{2} \geqslant 0$, and $C$ lies in an admissible twig of $D$, which is impossible by Lemma 2.8. Therefore, $T=\left[(2)_{k}\right]$, and $C$ does not lie in any rational twig of $D$, as claimed.

Our next lemma is a standard result concerning curves of canonical type, see eg. [Miy01, I.3.3.1]. It will give a description of the almost minimal model of $(X, D)$, or, more precisely, the pair obtained from $(X, D)$ after all possible half point attachments, called a strongly minimal model in [Miy01, II.4.9].

Recall that $\mathbb{F}_{m}$ for an integer $m \geqslant 0$ denotes the $m$-th Hirzebruch surface $\mathbb{P}\left(\mathcal{O}_{\mathbb{P}^{1}} \oplus \mathcal{O}_{\mathbb{P}^{1}}(m)\right)$.

Lemma 3.2 (strongly minimal models). Let $S$ be a smooth rational affine surface such that $\mathbb{C}[S]$ is a UFD. Let $(X, D)$ be a minimal $\log$ smooth completion of $S$. Assume that $K_{X}+D \equiv 0$ and that all $(-1)$-curves on $X$ are contained in $D$. Then one of the following holds:

(a) $X=\mathbb{F}_{m}$ for some $m \neq 1$ and $D=((0, m, 0,-m))$,

(b) $X=\mathbb{F}_{m}$ for some $m \neq 1$ and $D=((0, m,-m-2))$,

(c) $X=\mathbb{P}^{2}$ and $D=((-1,-1,-1))$, i.e. $D$ is a triangle,

(d) $X=\mathbb{P}^{2}$ and $D=((-1,-4))$, i.e. $D$ is a sum of a line and a conic which meet at two points.

Proof. Let $\tau:(X, D) \longrightarrow\left(X^{\prime}, D^{\prime}\right)$ be a contraction of some $(-1)$-curves in $D$ and its images, such that all singularities of $D^{\prime}$ are ordinary double points. Since $D$ is snc-minimal, $\operatorname{Bs} \tau^{-1} \subseteq \operatorname{Sing} D$. Let $A^{\prime}$ be a $(-1)$-curve in $X^{\prime}$. Then $A^{\prime} \cdot D^{\prime}=-A^{\prime} \cdot K_{X^{\prime}}=1$. If $A^{\prime} \nsubseteq D^{\prime}$ then $A^{\prime} \cap \operatorname{Sing} D^{\prime}=\emptyset$, so $\tau$ does not touch $A:=\tau_{*}^{-1} A$. Therefore, $A \subseteq X$ is a (-1)-curve not contained in $D$, contrary to our assumption. Hence $A^{\prime} \subseteq D^{\prime}$, so $\beta_{D^{\prime}}\left(A^{\prime}\right)=A^{\prime} \cdot D^{\prime}-A^{2}=2$. Since no two components of $D^{\prime}$ are tangent, $\# A^{\prime} \cap\left(D^{\prime}-A^{\prime}\right)=2$. After the contraction of $A^{\prime}$, the singularities of the image of $D^{\prime}$ are still at most ordinary double points.

Thus we can assume that $X^{\prime}$ has no $(-1)$-curves, i.e. $X^{\prime}=\mathbb{P}^{2}$ or $\mathbb{F}_{m}$ for some $m \neq 1$. By Lemma 2.1(a), the components of $D$ generate $\operatorname{Pic}(X)$, so the components of $D^{\prime}$ generate $\operatorname{Pic}\left(X^{\prime}\right)$, too.

Assume first that $X^{\prime}=\mathbb{P}^{2}$. Then $D^{\prime} \equiv-K_{\mathbb{P}^{2}} \equiv 3 \cdot$ [line]. Because the components of $D^{\prime}$ generate $\operatorname{Pic}\left(\mathbb{P}^{2}\right), D^{\prime}$ is reducible. In particular, $D^{\prime}$ has no singular component, so $\tau=$ id and $D$ is as in (c) or (d).

Assume now that $X^{\prime}=\mathbb{F}_{m}$. Then $2=\rho\left(X^{\prime}\right) \leqslant \# D^{\prime}$. Because $X^{\prime} \backslash D^{\prime}$ is affine, $D^{\prime}$ is connected, so $\beta_{D^{\prime}}(C) \geqslant 1$ for every component $C$ of $D^{\prime}$. Hence $0=C \cdot\left(K_{X^{\prime}}+D^{\prime}\right)=2 p_{a}(C)-2+\beta_{D^{\prime}}(C) \geqslant 2 p_{a}(C)-1$, It follows that $C$ is smooth, so $\tau=$ id. Moreover, $C$ is rational and $\beta_{D}(C)=2$, so $D$ is circular.

Denote by $F, T \in \operatorname{Pic}\left(\mathbb{F}_{m}\right)$ the classes of the fiber of $\mathbb{F}_{m}$ and of the section with $T^{2}=-m$, which is unique if $m \neq 0$. We have $F \cdot D=-F \cdot K_{\mathbb{F}_{m}}=2$, so by Lemma $2.4 D_{\text {hor }}$ consists of two 1-sections, say $H_{i} \equiv a_{i} F+T$ for $i \in\{1,2\}$. We can assume $a_{1} \geqslant a_{2}$. Note that either $a_{i}=0$ or $0 \leqslant H_{i} \cdot T=a_{i}-m$, so $a_{i} \geqslant m$. Hence $a_{1} \geqslant m$ and either $a_{2} \geqslant m$ or $H_{2} \equiv T$. Let $v$ be the number of fibers in $D$. Because all fibers are disjoint and meet $H_{1}$ and $H_{2}$, from the fact that $\beta_{D}\left(H_{j}\right)=2, j \in\{1,2\}$ we infer that

$$
2-v=H_{1} \cdot H_{2}=a_{1}+a_{2}-m .
$$

Suppose $v=0$. Then because $H_{1}, H_{2}$ generate $\operatorname{Pic}\left(\mathbb{F}_{m}\right)=\mathbb{Z}[F+T]$, we have $\operatorname{det}\left[\begin{array}{ll}a_{1} & 1 \\ a_{2} & 1\end{array}\right]= \pm 1$, so $a_{1}-a_{2}=1$. Now (3.1) gives $a_{2}=\frac{1}{2}(m+1)$, so $2 \nmid m$, hence $m \geqslant 3$ and $0 \neq a_{2}<m$, a contradiction

Thus $v \in\{1,2\}$. It follows that $H_{2} \equiv T$, because otherwise $a_{1}, a_{2} \geqslant m$ and (3.1) gives $1 \geqslant a_{1}+a_{2}-m \geqslant$ $m$, so $m=0$ and $1 \geqslant a_{1}+a_{2} \geqslant 2$, which is false. Hence $a_{2}=0, H_{2}^{2}=-m$ and by (3.1) $a_{1}=m+2-v$, so $H_{1}^{2}=m+4-2 v$. We conclude that (a) holds if $v=2$ and (b) holds if $v=1$.

Remark. Lemma 3.2 will imply that almost minimal models for surfaces in $\mathcal{S}_{0}$ are of type $(O)$ in [Fuj82, 8.70], cf. [Koj99, Proposition 1.5(1)]. For definition and basic properties of this type see [Fuj82, 8.8, 8.12]. Parts (a)-(d) correspond to cases 4, 6, 8 of 9 in [Koj99, Theorem 3.1]. We will not use these consequences. 
Proof of Theorem 1.1. Let $(X, D)$ be a minimal $\log$ smooth completion of $S \in \mathcal{S}_{0}$ and let

$$
\psi:(X, D) \longrightarrow\left(X_{n}, D_{n}\right)
$$

be an almost minimalization as in (2.2). By Proposition 2.14(d) $\kappa\left(X_{n} \backslash D_{n}\right)=\kappa(X \backslash D)=0$, so [Miy01, II.6.2.1] implies that $K_{X_{n}}+D_{n}^{\#} \equiv 0$, that is, $K_{X_{n}}+D_{n} \equiv \operatorname{Bk} D_{n}$. By Proposition 2.14(a) $\psi$ is a sequence of half-point attachments, so by Lemma 3.1(b) no base point of $\psi^{-1}$ lies on an admissible twig of $D_{n}$, i.e. $\operatorname{Bs} \psi^{-1} \cap \operatorname{Supp} \operatorname{Bk} D_{n}=\emptyset$. Hence $\psi_{*}^{-1} \operatorname{Bk} D_{n}=\psi^{*} \operatorname{Bk} D_{n} \equiv \psi^{*}\left(K_{X_{n}}+D_{n}\right)$, which by Lemma 2.1 writes uniquely as a sum of components of $D$, with integral coefficients. But $\psi_{*}^{-1} \mathrm{Bk} D_{n}$ is a sum of components of $D$ with coefficients in $[0,1)$. Hence $\psi_{*}^{-1} \mathrm{Bk} D_{n}=0$, which implies that

$$
K_{X_{n}}+D_{n} \equiv 0 \text {. }
$$

We claim that there is a sequence of half-point attachments

$$
\varphi:(X, D) \longrightarrow\left(Z, D_{Z}\right) \text { such that }\left(Z, D_{Z}\right) \text { is as in Lemma 3.2. }
$$

Indeed, let $\left(X_{n}, D_{n}\right) \longrightarrow\left(Z^{\prime}, D_{Z^{\prime}}\right)$ be any sequence of half-point attachments. By Remark 2.2, $\mathbb{C}\left[Z^{\prime} \backslash D_{Z}^{\prime}\right]$ is a UFD, and $K_{Z^{\prime}}+D_{Z^{\prime}} \equiv 0$ by (3.2). Let $A \subseteq Z^{\prime}$ be a (-1)-curve, $A \nsubseteq D_{Z^{\prime}}$. Then $A \cdot D_{Z^{\prime}}=-A \cdot K_{Z^{\prime}}=1$, so $A$ satisfies (2.1). After a half-point attachment of $A$, the image of $Z^{\prime}$ has smaller rank, so after finitely many half-point attachments, we get $\left(Z, D_{Z}\right)$ with no $(-1)$-curves off $D_{Z}$, as needed.

We need to show that, for a suitable $\log$ smooth completion $(X, D)$ of $S$, the morphism $\varphi$ from (3.3) can be chosen as in Construction 1.3. Denote by $A_{1}, \ldots, A_{r}$ the curves not contained in $D$ which are contracted by $\varphi$ (in particular, $r \geqslant n$ ). Write $\varphi\left(A_{i}\right)=\left\{b_{i}\right\} \subseteq Z$ for $i \in\{1, \ldots, r\}$. By Lemma 3.1(b) each $b_{i}$ lies on exactly one component $C_{i}$ of $D_{Z}$. Lemma 2.1 gives $\# D=\rho(X)$, so

$$
\# D_{Z}-\rho(Z)=\# D+r-\rho(X)=r,
$$

hence $r=2$ if $\left(Z, D_{Z}\right)$ is as in Lemma 3.2(a),(c) and $r=1$ if $\left(Z, D_{Z}\right)$ is as in Lemma 3.2(b),(d).

Let $R_{1}, \ldots, R_{r}$ be a basis of $\operatorname{ker}\left[\mathbb{Z}\left[D_{Z}\right] \longrightarrow \operatorname{Pic}(Z)\right]$, and let $a_{i j}$ be the coefficient of $C_{i}$ in $R_{j}$. We have $\operatorname{Pic}(X)=\operatorname{Pic}(Z) \oplus \mathbb{Z}[\operatorname{Exc} \varphi]=\mathbb{Z}\left[\varphi^{*} D_{Z}\right] /\left(\varphi^{*} R_{1}, \ldots, \varphi^{*} R_{i}\right)$. Because by Lemma 3.1(b) each $A_{i}$ has multiplicity 1 in $\varphi^{*} C_{i}$ and 0 in $\varphi^{*}\left(D_{Z}-C_{i}\right)$, Lemma 2.1(b) gives

$$
\operatorname{det}\left[a_{i j}\right]_{1 \leqslant i, j \leqslant r}= \pm 1 \text {. }
$$

Now we treat each of the cases (a)-(d) from Lemma 3.2 separately. We would like to reduce everything to case (a) with $m=0$. So in each of the remaining cases, we construct $\sigma: Z \rightarrow Z^{\prime}$, which is an isomorphism on $Z \backslash D_{Z}$ and on some neighborhood of $b_{1}, \ldots, b_{r}$; and $\varphi^{\prime \prime}:\left(Z^{\prime}, \sigma_{*} D_{Z}\right) \longrightarrow\left(Z^{\prime \prime}, D_{Z^{\prime \prime}}\right)$, which is an identity or a half-point attachment, such that $\left(D_{Z^{\prime \prime}}, D_{Z^{\prime \prime}}\right)$ is as in one of the cases not considered yet. Then we lift $\sigma$ so that the following diagram is commutative:

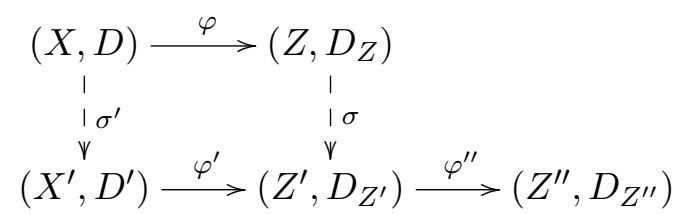

where $D^{\prime}, D_{Z^{\prime}}, D_{Z^{\prime \prime}}$ are reduced total transforms of $D$, and $\left(X^{\prime}, D^{\prime}\right)$ is another log smooth completion of $S$. Eventually, we replace $\varphi$ with $\varphi^{\prime \prime} \circ \varphi^{\prime}$, which is another sequence of half-point attachments.

Consider case (d), i.e. $r=1, D_{Z}$ is a sum of a line $L$ and a conic $C$, and $\# L \cap C=2$. We have $R_{1}=C-2 L$, so by (3.4) $b_{1} \in C$. Define $\sigma$ as a blow up at a point of $C \cap L$ followed by a flow (see Section 2D) on the proper transform of $L$. Then $\left(Z^{\prime}, D_{Z}^{\prime}\right)$ is as in case (b), so we put $\varphi^{\prime \prime}=$ id.

Consider case (c) and let $L \subseteq D_{Z}$ be the line not containing $b_{1}, b_{2}$. As before, we define $\sigma$ as a blowup at $L \cap\left(D_{Z}-L\right)$, followed by a flow on the proper transform of $L$. Then $\left(Z^{\prime}, D_{Z}^{\prime}\right)$ is as in case (a).

Consider case (b). Write $D_{Z}=F+T+H$, where $F^{2}=0, T^{2}=-m$ and $H^{2}=m+2$. We can assume that $b_{1} \in F$ : indeed, otherwise after $\pm C_{1}^{2}$ flows on $F$ we obtain a pair with that property. Now $r=1$ and $R_{1}=(m+1) F+T-H$, so (3.4) gives $m=0$. Let $\sigma$ be a flow on $T$ such that $\left(\sigma_{*} F\right)^{2}=1$, followed by a blowup at $\sigma(F \cap H)$. Let now $\varphi^{\prime \prime}$ be the contraction of a proper transform of a member of $|T|$ passing through $F \cap H$. The resulting pair is as in (a).

Eventually, consider case (a). Write $D_{Z}=F_{1}+T+F_{2}+H$, where $F_{1}^{2}=F_{2}^{2}=F_{1} \cdot F_{2}=0$ and as before $T^{2}=-m, b_{1}, b_{2} \notin T$. We have $r=2$ and $R_{1}=F_{1}-F_{2}, R_{2}=m F_{2}+T-H$, so (3.4) implies (after renaming $b_{i}, F_{i}$ if necessary) that $b_{1} \in F_{1}, b_{2} \in H+T$. After $m$ flows on $F_{2}$ we can assume that $m=0$, so $Z \cong \mathbb{P}^{1} \times \mathbb{P}^{1}$. We can choose coordinates on $Z$ such that $F_{1}=\ell_{1,0}, F_{2}=\ell_{1, \infty}, b_{2} \in H=\ell_{2,0}, T=\ell_{2, \infty}$, $b_{1}=(0,1), b_{2}=(1,0)$. Now by Lemma 3.1(b), $\varphi:(X, D) \longrightarrow\left(Z, D_{Z}\right)$ is as in Construction 1.3. 


\section{Diffeomorphism type of $S_{p_{1}, p_{2}}$}

In this section we prove Theorem 1.4. To this end, we need to translate Construction 1.3 to the language of Kirby calculus, where blowing up corresponds to attaching handles. We give a quick overview of handlebodies and Kirby diagrams in Section 4A. In Proposition 4.3 we show that Construction 1.3 amounts to attaching two 2-handles to $\mathbb{T}^{2} \times \mathbb{D}^{2}$, whose interior is identified with $\mathbb{C}^{*} \times \mathbb{C}^{*}$, see Figure 3 . Theorem 1.4 follows by sliding its 2-handle over the attached ones. The boundary $M$ of the obtained 4-manifold is a 0 -surgery on the knot $K$ from Figure 2. It is interesting to note that this is one of the few exceptional surgeries on $K$, classified in [BW01]. More precisely, while all but finitely many surgeries on $K$ are hyperbolic, $M$ contains incompressible tori. Decomposition of $M$ along these tori is described in Proposition 4.7. While interesting in its own right, it gives another way to distinguish $S_{p_{1}, p_{2}}$, for different $\left\{\operatorname{deg} p_{1}, \operatorname{deg} p_{2}\right\}$.

We begin with the following observation.

Proposition 4.1. For every $p_{1}, p_{2}, S_{p_{1}, p_{2}}$ is homotopically equivalent to $\mathbb{S}^{2}$.

Proof. Viewing $S=S_{p_{1}, p_{2}}$ as an iterated affine modification of $\mathbb{C}^{2}$, we infer from [Kal94, Lemma 3.4] that $\pi_{1}(S)=\pi_{1}\left(\mathbb{C}^{2}\right)=\{1\}$. This modification replaces $\left\{x_{1} x_{2}=0\right\} \subseteq \mathbb{C}^{2}$, which is contractible, by the affine part of $A_{1}+A_{2}$, which is a disjoint union of two copies of $\mathbb{C}^{1}$. Hence $e_{\text {top }}(S)=e_{\text {top }}\left(\mathbb{C}^{2}\right)-1+2=2$. Because $S$, being Stein, has a homotopy type of a CW-complex of (real) dimension two [GS99, 11.2.6], it follows that $H_{i}(S, \mathbb{Z})=\mathbb{Z}$ for $i=2$ and 0 for $i>2$. Thus $S$ has weak homotopy type of $\mathbb{S}^{2}$. Applying the Whitehead theorem to the continuous map $\mathbb{S}^{2} \longrightarrow S$ given by a generator of $\pi_{2}(S)=H_{2}(S ; \mathbb{Z})=\mathbb{Z}$, we infer that $\mathbb{S}^{2} \simeq_{\text {htp }} S$, as claimed.

\section{A. Overview of Kirby calculus}

We now briefly recall the language of Kirby diagrams of 4-manifolds, for a complete treatment see [GS99, §4-5]. A (4-dimensional) $k$-handle $h$ for $k \in\{0, \ldots, 4\}$ is a copy of $\mathbb{D}^{k} \times \mathbb{D}^{4-k}$ attached to a boundary of a 4-manifold $V$ via an embedding $\varphi: \partial \mathbb{D}^{k} \times \mathbb{D}^{4-k} \longrightarrow \partial V$. The images of $\partial \mathbb{D}^{k} \times 0$ and $0 \times \partial \mathbb{D}^{4-k}$ are called the attaching and belt spheres of $h$, the image of $\mathbb{D}^{k} \times 0$ is called the core of $h$, see [GS99, Fig. 4.1]. Smoothing corners gives a 4-manifold $V \cup_{\varphi} h$ which depends only on the attaching sphere of $h$ and, if $k=2$, on the integer called framing of $h$, which equals the linking number of its attaching circle and its push-off along a transverse vector field [GS99, 4.1, 4.5]. For example, blowing up is the same as attaching a 2-handle with framing -1 [GS99, p. 150].

Any 4-manifold $V$ can be obtained from $\mathbb{D}^{4}$ by attaching handles of increasing index. There are two ways of modifying such handle-decomposition without changing the diffeomorphism type of $V$. First, if the attaching sphere of a $k$-handle meets the belt sphere of a $(k-1)$-handle transversally in a single point, these two handles can be canceled [GS99, 4.2.9]. Second, a 2-handle $h$ can be slid over a 2-handle $h_{0}$ by pushing the attaching circle of $h$ through the belt circle of $h_{0}$ [GS99, 4.2.10]. We use the same letter to denote a handle before and after the slide.

A handle-decomposition of $V$ is encoded by a Kirby diagram, which is a (decorated) link in $\mathbb{S}^{3}$, i.e. in the boundary of the initial 0-handle. A 2-handle is represented by its attaching circle with a framing coefficient. Attaching a 1-handle to $V$ is the same as drilling a tubular neighborhood of a properly embedded $\mathbb{D}^{2} \subseteq V$ [GS99, p. 168], so it is represented by a circle $\partial \mathbb{D}^{2} \subseteq \partial V$, with a dot to distinguish it from a 2-handle. All dotted circles in a Kirby diagram form an unlink. Once 1- and 2-handles are attached, there is usually (e.g. if $V$ is closed or $\partial V$ is connected and $V$ is simply connected, which is our case) a unique way to attach the remaining ones [GS99, p. 148], so they are not drawn. We use the same letters for handles and corresponding knots in the diagram.

For example, a diagram consisting of one unknot with coefficient e represents a $\mathbb{D}^{2}$-bundle over $\mathbb{S}^{2}$ of Euler number $e$ [GS99, Fig. 4.20]. The diagram in Figure 3 without the leftmost and rightmost circle represents $\mathbb{T}^{2} \times \mathbb{D}^{2}$, see [GS99, Fig. 4.36]. Indeed, attaching the 1-handles gives $\left(\mathbb{T}^{2} \backslash\{\right.$ disk $\left.\}\right) \times \mathbb{D}^{2}$, whose fundamental group is freely generated by loops based in the initial 0-handle and going along the cores of the 1-handles. The 2-handle, attached along their commutator, caps off the puncture.

Handle cancellation and sliding is represented on the Kirby diagram as follows. First, if a dotted circle meets only one non-dotted circle, once, then the corresponding 1- and 2-handle cancel [GS99, Fig. 5.38]. Second, sliding a 2-handle $t$ over $t_{0}$ replaces $t$ by its band-sum with a parallel copy $\widetilde{t}_{0}$ of $t_{0}$ [GS99, Fig. 5.4]. More precisely, choose an arc in $t$ and $\widetilde{t}_{0}$ and connect their endpoints such that the obtained "square" bounds a band which is disjoint from the rest of the diagram. Recall that $\ell k\left(t_{0}, \widetilde{t}_{0}\right)$ is the framing of $t_{0}$. The new framing of $t$ is the sum of framings of $t$ and $t_{0}$ plus $2 \ell k\left(t, t_{0}\right)$ [GS99, p. 142]. It is important to remember that the orientations of $t$ and $\widetilde{t}_{0}$ should match. We denote by $-t$ the knot $t$ taken with opposite 
orientation. The components of a standard Hopf link drawn as in the left of Figure 7 are always meant to have counterclockwise orientation and linking number +1 .

\section{B. Handle decompositions and proof of Theorem 1.4}

Notation 4.2. Let $S=S_{p_{1}, p_{2}}$ be as in Construction 1.3. For $j \in\{1,2\}$ put $d_{j}=\operatorname{deg} p_{j}+1, T_{j, d_{j}}=L_{j, 0}$, and denote by $T_{j, i}, i \in\left\{1, \ldots, d_{j}-1\right\}$ the $i$-th component of the twig $T_{j}$ meeting $A_{j}$, see Figure 1 (a). Let $\operatorname{Tub}(D)$ be the tubular neighborhood of $D$ in $X$ constructed in [Mum61]. Put $M=\partial \operatorname{Tub}(D)$ as in Section $2 \mathrm{E}$ and $V=X \backslash \operatorname{int} \operatorname{Tub}(D)$. Clearly, $S \simeq_{\text {diffeo }}$ int $V$. We may and will assume $d_{1} \leqslant d_{2}$.

Proposition 4.3. The 4-manifold $V$ is obtained from $\mathbb{T}^{2} \times \mathbb{D}^{2}$ by attaching two 2-handles with framings $-d_{1},-d_{2}$ along the standard generators of $\pi_{1}\left(\mathbb{T}^{2}\right)$. Its Kirby diagram is given by Figure 3.

Proof. Let $\varphi$ be as in Construction 1.3. Let $\mathbb{D} \subseteq \mathbb{C}^{2} \subseteq \mathbb{P}^{1} \times \mathbb{P}^{1}$ be a closed disk of radius 2 centered at $(0,0)$, so that the base points $(0,1),(1,0)$ of $\varphi^{-1}$ belong to int $\mathbb{D}$. Put $X_{0}:=\varphi^{-1}(\mathbb{D}) \subseteq X, \varphi_{0}:=\left.\varphi\right|_{X_{0}}: X_{0} \longrightarrow \mathbb{D}$ and $\ell_{i, j}^{\prime}=\ell_{i, j} \cap \mathbb{D}$. Figure 6 shows $\partial \varphi_{0}^{-1}\left(\ell_{1,0}^{\prime} \cup \ell_{2,0}^{\prime}\right)$, where the attaching circles of the 2 -handles are drawn together with their framings.

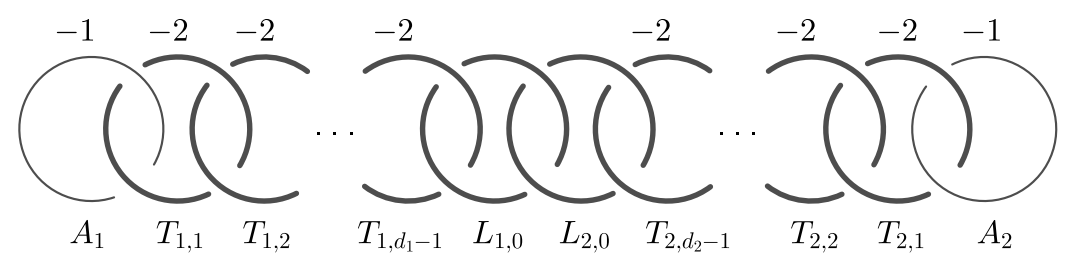

FiguRE 6 . Underlying disks of $L_{1,0}, L_{2,0}$ and handles attached to $\mathbb{C}^{2}$ by $\varphi_{0}$.

To construct $V$, we need to remove from $X_{0}$ a tubular neighborhood of $\varphi_{0}^{-1}\left(\ell_{1,0}^{\prime} \cup \ell_{2,0}^{\prime}\right) \backslash\left(A_{1} \cup A_{2}\right)$. To do this, we first remove from the 0-handle all disks whose boundaries are drawn in Figure 6 in bold, and then we remove the cores of the corresponding 2-handles (everything suitably thickened).

By [GS99, p. 214], the first step gives a Kirby diagram obtained from Figure 6 as follows. Replace each circle corresponding to $T_{j, i}$ for $j \in\{1,2\}, i \in\left\{1, \ldots, d_{j}\right\}$ by a dotted circle $t_{j, i}^{\prime}$, and each twist corresponding to $T_{j, i+1} \cap T_{j, i}$ (resp. $L_{0,1} \cap L_{0,2}$ ) by a 0 -framed 2-handle $h_{j, i}$ (resp. $h$ ), linked with the dotted circles as in Figure 7 . We denote by $a_{j}$ the 2-handle corresponding to $A_{j}$.

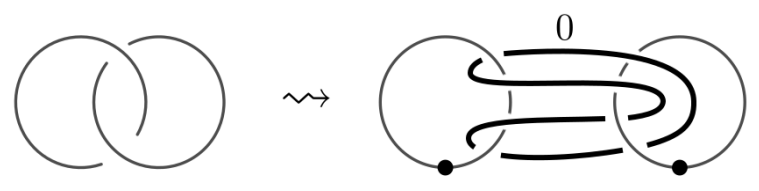

FiguRE 7. Intersection of two removed disks, see [GS99, Fig. 6.27].

By [GS99, p. 224], the next step amounts to attaching a 2-handle along a parallel copy of each removed circle, together with a certain 3-handle. In our case, for each $T_{j, i}, j \in\{1,2\}, i \in\left\{1, \ldots, d_{j}-1\right\}$ we attach a (-2)-framed 2-handle $t_{j, i}$ such that $\ell k\left(t_{j, i}, t_{j, i}^{\prime}\right)=-2$ and $\ell k\left(t_{j, i}, t_{j, i+1}^{\prime}\right)=\ell k\left(t_{j, i}, t_{j, i-1}^{\prime}\right)=1$, where we put $t_{j, 0}^{\prime}=a_{j}$. The resulting Kirby diagram is shown in Figure 8 (where we only draw the part corresponding to $L_{1,0}+L_{2,0}+T_{2}+A_{2}$ and skip the subscript "2,": the other part is analogous).
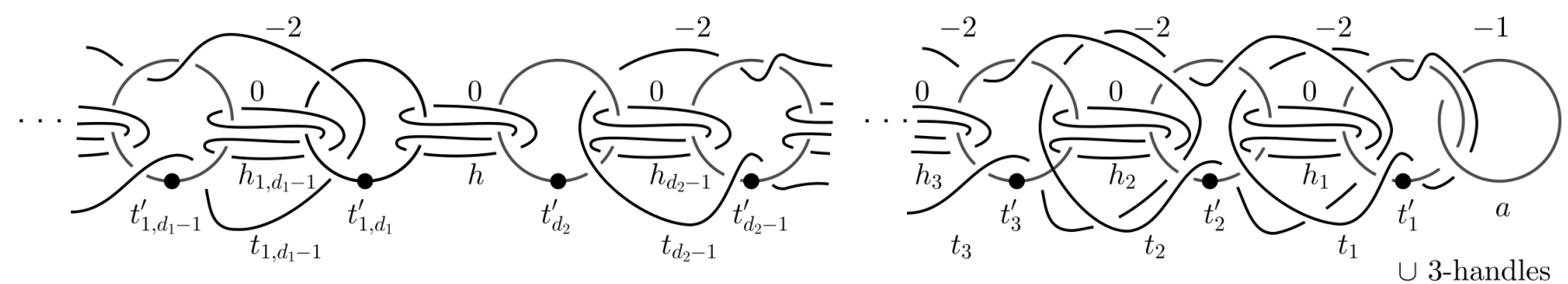

Figure 8. Right half of the Kirby diagram for $V=X \backslash \operatorname{Tub}(D)$.

We will simplify this diagram by handle slides. First, we slide $t_{j, 1}$ over $a_{j}$, see Figure 9 . After this slide, $t_{j, 1}$ unlinks from $a$, meets $t_{j, 1}^{\prime}$ once with $\ell k\left(t_{j, 1}, t_{j, 1}^{\prime}\right)=-1$ and changes framing to -1 , while the rest of 

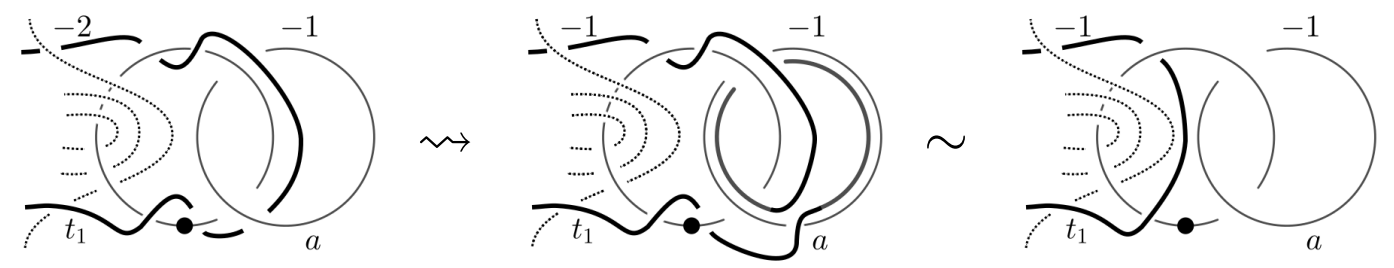

FIGURE 9. Sliding $t_{j, 1}$ over $a_{j}$.

the diagram does not change. Applying Lemma 4.4(a) below inductively to pairs $\left(t_{0}, t\right)=\left(t_{j, i}, t_{j, i+1}\right)$ for $i=1, \ldots, d_{j}-1$, we obtain a diagram in Figure 10 .

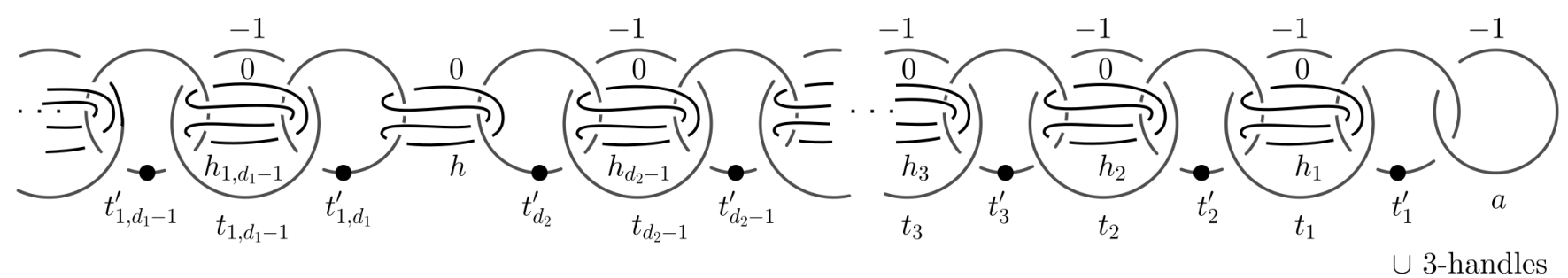

FiguRE 10. Right half of the Kirby diagram after sliding $t_{i}$ over $t_{i-1}$ for $i=1,2, \ldots, d_{2}-1$

Now by Lemma 4.4(b) each $h_{i, j}$ can be slid to a 0 -framed unknot disjoint from the rest of the diagram. Since $\pi_{1}(V)=\{1\}$ by Proposition 4.1, [GS99, p. 148] shows that all $h_{j, i}$ 's cancel with all 3-handles (recall that there are $d_{1}+d_{2}-2$ of them, attached while removing the cores of $T_{j, i}$ 's). Applying Lemma 4.4(c) inductively to pairs $(a, t)=\left(t_{j, i}, t_{j, i+1}\right), i=1, \ldots, d_{j}-1$ gives Figure 3 .

In the above proof, we used the following standard exercise in handle slides, cf. [GS99, p. 142].

Lemma 4.4. Let $\ell_{1}, \ell_{2}$ be links in the left and right of one of the Figures 11(a)-(c). Define $\ell \subseteq \ell_{1}$ as $t_{0}$ in (a), $t+h$ in (b) and $t+c_{0}+a$ in (c). Let $k$ be a Kirby diagram containing $\ell_{1}$ in such a way that $\ell$ is disjoint from $k-\ell_{1}$. Then $k$ is equivalent to a diagram obtained from $k$ by replacing $\ell_{1}$ with $\ell_{2}$.

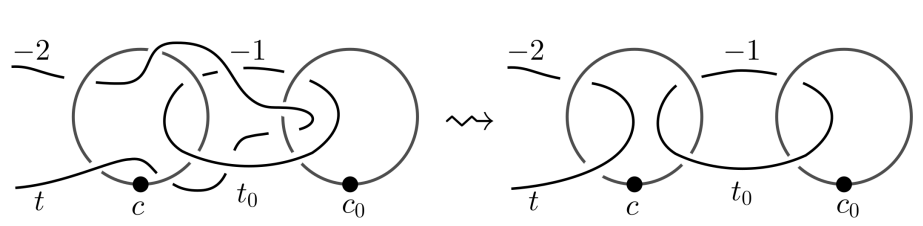

(A) Sliding $t$ over $t_{0}$

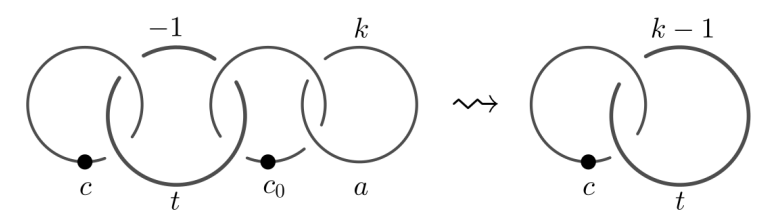

(C) Sliding $t$ over $a$ and canceling $\left(a, c_{0}\right)$

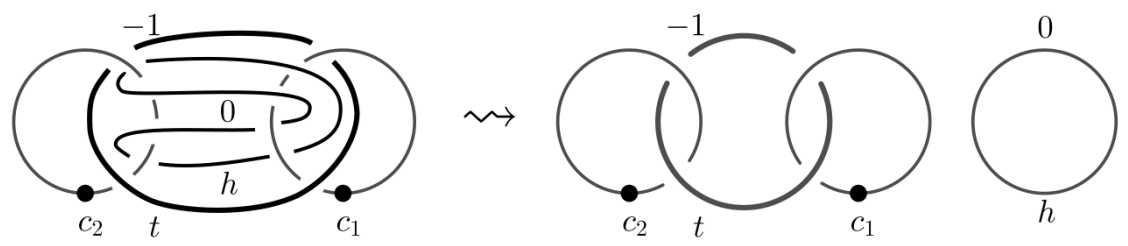

(B) After adding and subtracting $t$, a 2-handle $h$ like in Figure 7 is ready to be canceled with a 3-handle

Figure 11. Lemma 4.4: handle slides applied inductively in the proof of Proposition 4.3

Proof. (a) In order to have a clear picture, we draw $t_{0}$ rotated around a horizontal line, so that both $t_{0}$ and $t$ go under the bottom part of $c_{0}$, see Figure 12(a). We draw a parallel copy of $t_{0}$ in such a way that it twists over $t_{0}$ near that crossing, and cut this twist to make a band-sum with $t$. Now the part of $t$ on the right of the vertical diameter of $c$ can be isotoped to the latter, which proves the claim.

(b) As in (a), we draw $t$ rotated so that both $h$ and $t$ go under the bottom part of $c_{2}$, and do the band-sum there, see Figure 12(b). Then the bottom part of $h$ untangles from $c_{1}, c_{2}$, and becomes a parallel copy of $t$. Sliding it over $-t$ unlinks it from the rest of the diagram.

(c) After sliding $t$ over $a$, it becomes disjoint from $c_{0}$ and meets $c$ as before, see Figure 12(c), where the box stands for $k$ twists. Moreover, $c_{0}$ now meets only $a$, once, so these handles cancel. 

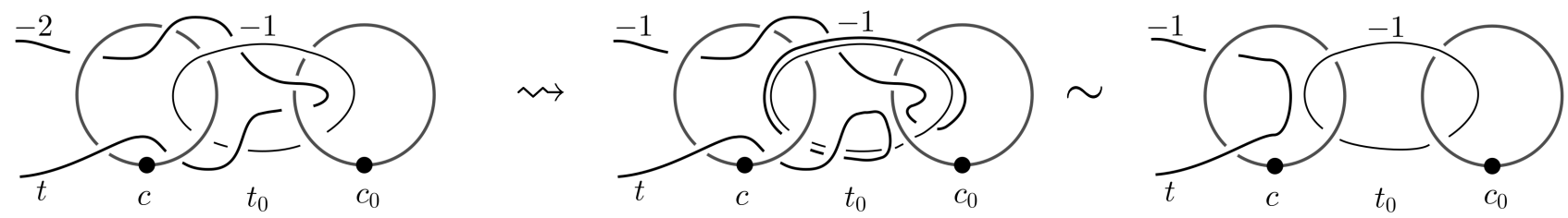

(A) Sliding $t$ over $t_{0}$
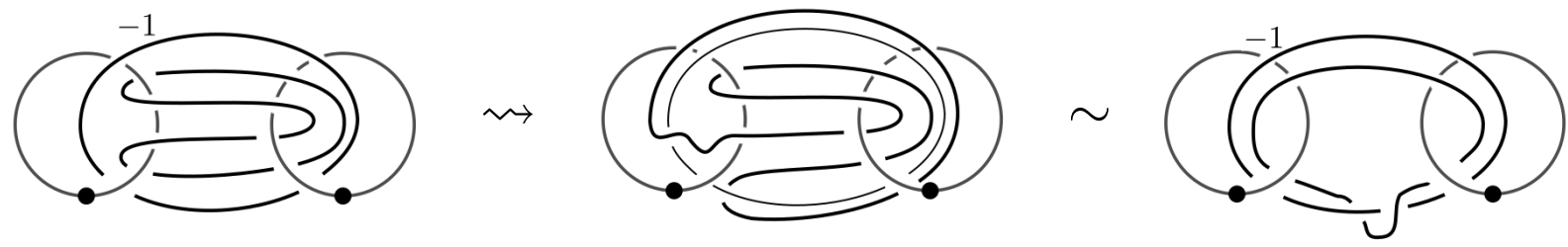

(B) After sliding over $t, h$ becomes its parallel copy.
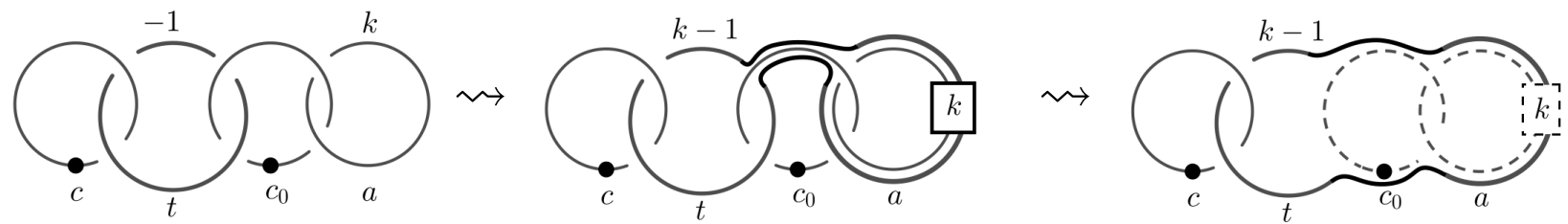

(C) Canceling the rightmost 1/2-handle pair.

Figure 12. Proof of Lemma 4.4.

Proof of Theorem 1.4. Recall that by Proposition 4.3, $S$ is diffeomorphic to an interior of a 4-manifold with Kirby diagram as in Figure 3, and by Corollary 2.10 such $S$ 's are not homeomorphic for different $\left\{d_{1}, d_{2}\right\}$. Therefore, it remains to prove that Kirby diagrams in Figures 3 and 2 are equivalent.
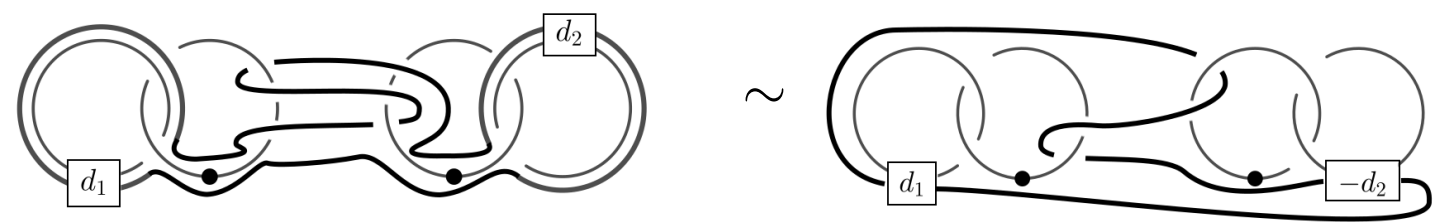

(A) Adding $a_{2}-a_{1}$ to $h$.
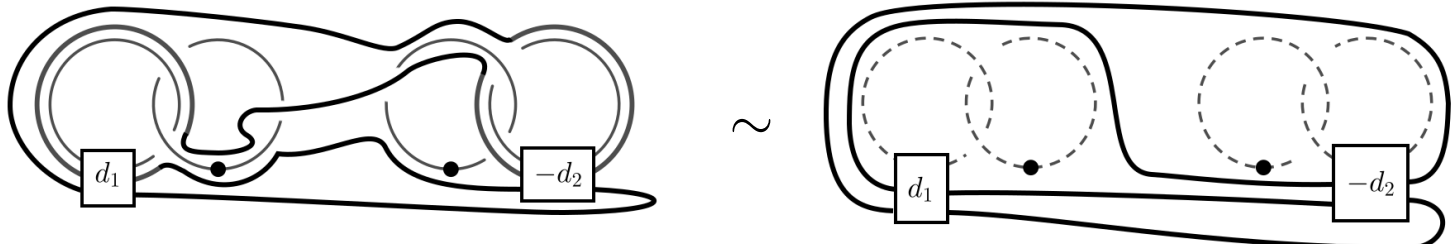

(B) Subtracting $a_{2}-a_{1}$ from $h$ and canceling pairs $\left(c_{j}, a_{j}\right)$.

Figure 13. Proof of Theorem 1.4

Let $h, a_{j}$ be the 0 - and $\left(-d_{j}\right)$-framed 2-handles in Figure 3, and let $c_{j}$ be the 1-handle meeting $a_{j}$. We will add and subtract $a_{2}-a_{1}$ from $h$ so that the latter untangles from $c_{1}, c_{2}$. Then we cancel pairs $\left(c_{j}, a_{j}\right)$. This is done in Figure 13, which we now explain; see also [GS99, 5.1.8(b)] for the case $d_{1}=d_{2}=1$.

We start with Figure 3, but draw it with $a_{2}$ rotated along a horizontal axis. Next, we draw parallel copies of $-a_{1}$ and $a_{2}$, where a box stands for $d_{j}$ twists. Then we band sum $h$ with those copies near the points where $h$ and $a_{j}$ go under $c_{j}$, see Figure 13(a). This move resolves nearby crossings of $h$ and $c_{j}$, leaving $\ell k\left(h, c_{j}\right)=-1$. Now we rotate $a_{2}$ again (this changes the sign in the box) and repeat the above move, but with reversed orientation of $a_{j}$, i.e. we add $a_{1}$ and subtract $a_{2}$. As before, the crossings of $h$ with $c_{j}$ get resolved, so these 1-handles cancel with $a_{j}$, see Figure 13(b). After the first slide, $h$ twisted along $a_{j}$ in the boxes, so now it twists there over itself. A look at Figure 13(b) shows that we do obtain a knot as in Figure 2. The framing of $h$ remains 0 since we added and subtracted the same thing. 


\section{C. Computation of $\pi_{1}^{\infty}\left(S_{p_{1}, p_{2}}\right)$ and a relation with knot surgeries}

Proposition 4.3 allows us to compute the fundamental group of $V$ (which is trivial by Proposition 4.1), and, more interestingly, that of $M$, which equals $\pi_{1}^{\infty}(S)$, see [Mum61].

Proposition 4.5. The fundamental group at infinity of $S$ has a presentation

$$
\pi_{1}^{\infty}(S)=\left\langle\delta_{1}, \delta_{2}, \lambda \mid \delta_{1}=\left[\gamma_{2}, \lambda^{-1}\right], \delta_{2}=\left[\gamma_{1}, \lambda\right],\left[\gamma_{1}, \gamma_{2}\right]=1\right\rangle, \quad \text { where } \gamma_{j}=\delta_{j}^{d_{j}} .
$$

Moreover, $\gamma_{j}$ for $j \in\{1,2\}$ is the attaching circle of the $\left(-d_{j}\right)$-framed 2-handle in Figure 3 , and $\lambda$ is the belt sphere of the 0 -framed one.

Proof. Let $\gamma_{1}, \gamma_{2}, \lambda \in \pi_{1}\left(\partial\left(\mathbb{T}^{2} \times \mathbb{D}^{2}\right)\right)=\pi_{1}\left(\mathbb{T}^{3}\right)$ be the standard generators. Proposition 4.3 shows that $M$ is obtained from $\mathbb{T}^{3}$ by a surgery along $\gamma_{1}, \gamma_{2}$ with coefficients $d_{1}, d_{2}$. The meridians of deleted tori are $\mu_{1}:=\left[\gamma_{2}, \lambda^{-1}\right], \mu_{2}:=\left[\gamma_{1}, \lambda\right]$. Now by van Kampen theorem, each surgery replaces the relation $\mu_{j}=1$ in $\pi_{1}\left(\mathbb{T}^{3}\right)$ by $\mu_{j}=\delta_{j}$, where $\delta_{j} \in \pi_{1}(M)$ is the corresponding attaching circle, satisfying $\delta_{j}^{d_{j}}=\gamma_{j}$.

Remark 4.6. The presentation (4.1) can also be obtained from the plumbing construction in [Mum61], see Section 2E. If $D$ is a tree, [Mum61] shows how to calculate $\pi_{1}(M)$ in terms of fibers of the plumbed $\mathbb{S}^{1}$ bundles, using van Kampen theorem. For our $D$, one needs to use the van Kampen theorem for groupoids [Bro67], say, to glue $\partial \operatorname{Tub}\left(L_{1, \infty}+L_{2, \infty}\right)$ to the rest of $\partial \operatorname{Tub}(D)$ along two disjoint plumbing tori, each containing a base point. This gives the additional generator $\lambda$, which is the sum of paths joining the two base points in each of the glued pieces. In this interpretation, $\gamma_{j}$ is the fiber over $L_{j, 0}$. Indeed, in the proof of Proposition 4.3 it was drawn parallel to the boundary of a disk corresponding to $T_{j, d_{j}-1}$, which goes once around $L_{j, 0}$. In turn, $\delta_{j}$ is a fiber over $T_{j, 1}$, that is, a singular fiber of a lens space $L\left(1, d_{j}\right)$ attached to $\partial \operatorname{Tub}\left(L_{j, 0}\right)$.

In the proof of Corollary 2.10, we have essentially seen the JSJ decomposition of $M$. We will now show how to see it from the above description of $M$, as a 0 -surgery on $K_{\left[2 d_{1}, 2 d_{2}\right]}$. In order to settle the notation, we recall the basic definitions following [Hat07].

Let $N$ be a 3-manifold. A surface $\Sigma$ in $N$ is incompressible if every loop in $\Sigma$ bounding a disk in $N$ bounds a disk in $\Sigma$, too. We write $N \mid \Sigma$ for a 3-manifold (with boundary) obtained from $N$ by removing $\Sigma$ together with some tubular neighborhood. Assume that $N$ is irreducible (see Section $2 \mathrm{E}$ ). Then $N$ has a collection $\Sigma$ of incompressible tori such that each component of $N \mid \Sigma$ is either a Seifert fiber space or has no incompressible tori. A minimal such $\Sigma$ (in the sense of inclusion) is unique up to an isotopy [Hat07, Theorem 1.9], and $N \mid \Sigma$ is called a JSJ-decomposition of $N$.

We now recall the notation for Seifert fibered spaces following [Hat07, §2.1]. Let $B$ be a compact surface of genus $g$ with $r$ boundary components, and let $M_{0} \longrightarrow B_{0}$ be an $\mathbb{S}^{1}$-bundle, where $B_{0}$ is $B$ with $k$ disks $D_{1}, \ldots, D_{k}$ removed. The preimage $T_{i} \subseteq M_{0}$ of $\partial D_{i}$ is a torus: we attach to it a solid torus $\mathbb{D}^{2} \times \mathbb{S}^{1}$ in such a way that a meridian $0 \times \mathbb{S}^{1}$ is attached to a loop in $T_{i}$ whose preimage in its universal cover is a line of slope $\frac{p_{i}}{q_{i}}$. The resulting manifold is a Seifert fibered space $M\left(g, r ; \frac{p_{1}}{q_{1}}, \ldots, \frac{p_{k}}{q_{k}}\right)$. It is a graph manifold. Its graph has one vertex corresponding to $M_{0}$ and $k$ maximal twigs, whose type is a continued fraction expansion of $\frac{p_{i}}{q_{i}}$. This graph is normal whenever a Seifert fibration is unique, see [Neu81, 5.7]. Few important exceptions, including lens spaces $\mathbb{S}^{3} / \mathbb{Z}_{p}$, are listed in [Hat07, Theorem 2.3]. Their normal graphs are described in [Neu81, 6.1].

Let again $M=\partial \operatorname{Tub}(D)$ be as in Notation 4.2. The following result can be inferred from the computation of $\Gamma(M)$ in Figure 4 using the algorithm in [Neu81, §5]. Nonetheless, we sketch a direct argument using the geometric description of incompressible tori in $M$ deduced in [BW01, Lemma 2.2] from [HT85].

Proposition 4.7 (JSJ decomposition of $M$ ). Let $\Sigma_{1}, \Sigma_{2} \subseteq M$ be the plumbing tori at $L_{1,0} \cap L_{2,0}$ and $L_{1,0} \cap L_{2, \infty}$, see Section 2E. Then the JSJ decomposition of $M$ is

(a) $M \mid\left(\Sigma_{1} \sqcup \Sigma_{2}\right)=M\left(2,0 ; \frac{1}{d_{1}}\right) \sqcup M\left(2,0 ; \frac{1}{d_{2}}\right)$ if $d_{1}, d_{2} \neq 1$,

(b) $M \mid \Sigma_{1}=M\left(2,0 ; \frac{1}{d_{2}}\right)$ if $d_{1}=1$ and $d_{2}>1$,

(c) $M=M\left(0,0 ; \frac{1}{2}, \frac{1}{3}, \frac{1}{6}\right)$ if $d_{1}=d_{2}=1$.

Let $\gamma_{j}, \delta_{j}$ be as in Proposition 4.5. Then $\delta_{j}$ is the singular fiber of $M\left(2,0 ; \frac{1}{d_{j}}\right)$ and $\gamma_{j}$ is a general one.

Proof. By Theorem 1.4, $M$ is a 0-surgery on $\mathbb{S}^{3}$ along $K=K_{\left[2 d_{1}, 2 d_{2}\right]}$. Because $K_{[2,2]}$ is just a trefoil, (c) follows from [Mos71, Proposition 3.1] which completely describes surgeries on torus knots. Assume $d_{2}>1$. If $d_{1}>1$, too, then cutting along $\Sigma_{1} \sqcup \Sigma_{2}$ in case (a) corresponds to removing the edges of the circular subgraph in Figure 4(b), and decorating the leftmost vertices with $r=2$, i.e. puncturing the 
base spheres twice. If $d_{1}=1$ then cutting along $\Sigma_{1}$ corresponds to removing the loop in Figure 4 (c) and adding boundary as before. This shows the equalities in (a)-(b), and proves that $M$ and, if $d_{1}=1, M \mid \Sigma_{i}$ for $i \in\{1,2\}$ are not Seifert fibered: indeed, $\Gamma\left(M \mid \Sigma_{i}\right)$ is not one of the graphs in [Neu81, 5.7, 6.1]. It remains to show that $\Sigma_{1}, \Sigma_{2} \subseteq M$ are incompressible.

The 3-manifold $M$ is irreducible by [HT85, Theorem 2] (it also follows from Lemma 2.9(b)). Put $M_{0}=\mathbb{S}^{3} \backslash \operatorname{Tub}(K) \subseteq M$. Put $M_{0}=\mathbb{S}^{3} \backslash \operatorname{Tub}(K) \subseteq M$. By [HT85, Proposition 1(1) and the proof of Proposition 2] there are, up to isotopy, at most two incompressible surfaces $\Sigma_{0} \subseteq M_{0}$ such that the surfaces $\Sigma \subseteq M$ obtained by capping $\Sigma_{0}$ are tori (see also proof of [BW01, Lemma 2.2]). Such $\Sigma_{0}$ are denoted by $S_{1}(0)$ and $S_{1}(1)$ in [HT85], and they have exactly one boundary component each. By [Prz83, Proposition 1.5(a)] they are unknotted (see Definition 1.3 loc. cit), so by Theorem 1.4 loc. cit, $\Sigma$ remains incompressible. Moreover, by [HT85, Theorem 1(e)] $S_{1}(0)$ is isotopic to $S_{1}(1)$ if and only if $d_{1}=1$. Thus our claim will follow once we show that $\Sigma=\Sigma_{1}, \Sigma_{2}$ for $\Sigma_{0}=S_{1}(0), S_{1}(1)$.

To this end, we recall the description of $S_{1}(0), S_{0}(1)$ from [HT85, p. 227, Fig. 1]. Consider Figure 2 as a knot in $\mathbb{R}^{3}$, and let $p$ be a vertical plane between the "twists". It meets the knot in four points. Connect them to get a quadrilateral $q \subseteq p$ : it is a 0 -handle for $S_{1}(0)$. Now attach to it two 1-handles along the bands with $d_{1}$ and $d_{2}$ twists, respectively: this gives a punctured torus $S_{1}(0)$. The torus $S_{1}(1)$ is constructed the same way, with $q$ replaced by its complement in the compactification $\bar{R}=\mathbb{S}^{2} \times\{\mathrm{pt}\} \subseteq$ $\left(\mathbb{S}^{2} \times \mathbb{R}\right) \sqcup\{ \pm \infty\}=\mathbb{S}^{3}$.

The loops based at $q$ and going around the 1-handles of $S_{1}(0)$ are isotopic to the non-dotted circles in Figure 3, which by Remark 4.6 are fibers $\gamma_{j}$ over $L_{j, 0}$. Hence $S_{1}(0)$ caps to $\Sigma_{1}$. To see the 1 -handles of $S_{1}(1)$, we need to change the base point from $q$ to its complement. The gluing described in Remark 4.6 shows that this amounts to changing, say, $L_{2,0}$ to $L_{2, \infty}$. This way we get $\Sigma_{2}$, as claimed.

Remark 4.8 (A direct argument for minimality of $\Sigma$ ). In the above proof, we have used the classification of graph manifolds to show that the collections $\Sigma_{1} \sqcup \Sigma_{2}$ in (a) and $\Sigma_{1}$ in (b) of incompressible tori decomposing $M$ to Seifert fibered spaces is minimal. However, this can be also seen directly, as follows.

First, we note that since $\Gamma\left(M \mid \Sigma_{i}\right)$ is a tree, one can use van Kampen theorem as in [Mum61] to compute

$$
\pi_{1}\left(M \mid \Sigma_{1}\right)=\left\langle\delta_{1}, \delta_{2} \mid\left[\gamma_{1}, \gamma_{2}\right]=1\right\rangle \text {, where } \delta_{j} \text { and } \gamma_{j}=\delta_{j}^{d_{j}} \text { are as in Remark 4.6. }
$$

Suppose that $d_{1}, d_{2}>1$, but $M \mid \Sigma_{i}$ is Seifert fibered for some $i \in\{1,2\}$. Then by [Hat07, Proposition 1.11], the incompressible torus $\Sigma_{3-i} \subseteq M \mid \Sigma_{i}$ is either horizontal or vertical. In the first case, it covers the base orbifold, which has two boundary components, so comparing Euler characteristics as done in [Hat07, p. 22] shows that $M \mid \Sigma_{i}$ has no singular fibers, so $M \mid \Sigma_{i} \cong\left(\mathbb{D}^{1} \times \mathbb{S}^{1}\right) \times \mathbb{S}^{1}$, a contradiction with (4.2). In the second case, cutting $M \mid \Sigma_{i}$ further along $\Sigma_{3-i}$ preserves the Seifert fibration: therefore by the equality in (a), it restricts to the unique one on $M\left(2,0 ; \frac{1}{d_{j}}\right)$. Hence after gluing $\Sigma_{3-i}$ back, the fiber of one fibration becomes a loop around the degenerate fiber of the other. This is a contradiction, because the former is central in $\pi_{1}$, and the latter is not.

Suppose now that $d_{2}>1$, but $M$ is Seifert fibered. Again, the incompressible torus $\Sigma_{1}$ is either horizontal or vertical. In the first case, since $M \mid \Sigma_{1}$ is connected, [Hat07, p. 22] implies that $M \mid \Sigma_{1} \cong$ $\Sigma_{1} \times \mathbb{D}^{1}$, a contradiction with (4.2). In the second case, we obtain that $M \mid \Sigma_{1}$ is a Seifert fiber space. We have shown that this is possible only if $d_{1}=1$, in which case the general fiber is represented by $\gamma_{1} \in \pi_{1}(M)$. In particular, $\left\langle\gamma_{1}\right\rangle \subseteq \pi_{1}(M)$ is a (nontrivial) central subgroup. Hence by (4.1) $\delta_{2}=\left[\gamma_{1}, \lambda\right]=1$ and $\delta_{1}=\left[\delta_{2}^{d_{2}}, \lambda^{-1}\right]=1$, so $\gamma_{1}=1$; a contradiction.

\section{5. $\mathbb{C}^{*}$-FIBRATions AND COMPlete ALGEBRAiC VECTOR FIELDS ON $S_{p_{1}, p_{2}}$}

In this section we prove Theorem 1.6(b). It follows from [KKL20, Theorem 1.6] that a flow of any complete algebraic vector field on $S_{p_{1}, p_{2}}$ preserves some $\mathbb{C}^{*}$-fibration of $S_{p_{1}, p_{2}}$ (see Proposition 5.5(c) for details). Therefore, to understand AAuthol $\left(S_{p_{1}, p_{2}}\right)$ we need to classify all $\mathbb{C}^{*}$-fibrations of $S_{p_{1}, p_{2}}$.

Their degenerate fibers are affine curves with Euler characteristic at least $e_{\text {top }}\left(\mathbb{C}^{*}\right)$, see [Miy01, III.1.8], i.e. unions of $\mathbb{C}^{*}$ and $\mathbb{C}^{1}$. Therefore, we begin by classifying all curves in $S_{p_{1}, p_{2}}$ which are isomorphic to $\mathbb{C}^{1}$. This is done in Lemma 5.2. In Lemma 5.3 we show that any $\mathbb{C}^{*}$-fibration of $S_{p_{1}, p_{2}}$ comes from a $\mathbb{C}^{*}$-fibration of a complement of suitably chosen pair of disjoint $\mathbb{C}^{1}$ 's; which by Lemma 5.4 turns out to be a torus $\mathbb{C}^{*} \times \mathbb{C}^{*}$. The union of such tori is exactly the complement of $Z$ from Theorem 1.6(b).

Notation 5.1. As in Notation 4.2, we put $d_{j}=\operatorname{deg} p_{j}+1$ and write $S:=S_{p_{1}, p_{2}}$. We use notation introduced in Construction 1.3 and denote by $T_{j}$ the maximal (-2)-twig of $D$ meeting $A_{j}$. Additionally, we denote by $c=\left\{x_{1}+x_{2}=1\right\} \subseteq \mathbb{P}^{1} \times \mathbb{P}^{1}$ the curve of type $(1,1)$ passing through $(\infty, \infty)$ and both base points $(1,0),(0,1)$ of $\varphi^{-1}$, and by $C:=\varphi_{*}^{-1} c$ its proper transform on $X$. 
It would be sometimes convenient to work with explicit equations of $S$. To write them, denote by $\widehat{p}_{j}=t^{d_{j}-1} p_{j}\left(t^{-1}\right), j \in\{1,2\}$ the polynomial obtained from $p_{j}$ by writing its coefficients in the opposite order. Note that $\widehat{p}_{j}(0)=1$ because $p_{j}$ is monic. Now $S \subseteq \mathbb{C}^{4}=\operatorname{Spec} \mathbb{C}\left[x_{1}, x_{2}, y_{1}, y_{2}\right]$ is given by

$$
y_{1} x_{1}^{d_{1}}=x_{2}-\widehat{p}_{1}\left(x_{1}\right), \quad y_{2} x_{2}^{d_{2}}=x_{1}-\widehat{p}_{2}\left(x_{2}\right) .
$$

Note that $S$ is in fact a surface in $\mathbb{C}^{3}$ because, say, $x_{1}$ can be computed from the second equation.

Lemma $5.2\left(\mathbb{C}^{1}\right.$ 's in $S$, cf. [KK11, Lemma 8.1]). A curve $\Gamma \subseteq X$ satisfies $\Gamma \cap S \cong \mathbb{C}^{1}$ if and only if one of the following holds:

(a) $\Gamma=A_{j}$ for some $j \in\{1,2\}$. Then $\Gamma^{2}=-1$ and $\Gamma \cdot D=\Gamma \cdot T_{j}=1$.

(b) $\Gamma=L_{j, 1}$ and $p_{j}=t^{d_{j}-1}$ for some $j \in\{1,2\}$. Then $\Gamma^{2}=-d_{j}, \Gamma \cdot D=\Gamma \cdot L_{3-j, \infty}=1$ and $\Gamma \cdot A_{3-j}=1$.

(c) $\Gamma=C$ and $p_{j}=1$ or $t^{d_{j}-2}(t-1)$ for both $j \in\{1,2\}$. Then $\Gamma^{2}=2-d_{1}-d_{2}, \Gamma \cdot D=2$, $\Gamma$ meets $D$ at $L_{1, \infty} \cap L_{2, \infty}$ and $\Gamma \cdot A_{j}=1$ for $j \in\{1,2\}$.

Moreover, $A_{1} \cdot A_{2}=0, L_{1,1} \cdot L_{2,1}=1$ and $A_{j} \cdot L_{j, 1}=C \cdot L_{j, 1}=0$ for $j \in\{1,2\}$.

Proof. Clearly, $A_{j} \cap S \cong \mathbb{C}^{1}$ and $A_{j}^{2}=-1$ as in (a). We will now check when $L_{j, 1} \cap S \cong \mathbb{C}^{1}$. Because $\ell_{j, 1}$ meets $\varphi_{*} D$ transversally on $\ell_{3-j, 0}$ and $\ell_{3-j, \infty}$, we have $L_{j, 1} \cdot\left(D+A_{3-j}\right)=2$ and $L_{j, 1}$ meets $L_{3-j, \infty}$, so $L_{j, 1} \cap S \cong \mathbb{C}^{1}$ if and only if $L_{j, 1}$ meets $A_{3-j}$, that is, all $d_{j}$ blowups over $\ell_{j, 1} \cap \ell_{3-j, 0}$ touch the image of $L_{j, 1}$ (hence $L_{j, 1}^{2}=\ell_{j, 1}^{2}-d_{j}=-d_{j}$ ). The proper transform of $\ell_{j, 1}$ after the first blowup meets the exceptional curve at the point with coordinate $\left(x_{3-j}-1\right) / x_{j}=0$ (see Construction 1.3), so the next blowup, if occurs, touches it if and only if the coefficient near $t^{d_{j}-2}$ in $p_{j}$ is zero. Because these two curves meet transversally, the infinitely near points on the next proper transforms will have coordinate zero, too. Following Construction 1.3 we conclude that $L_{j, 1} \cap S \cong \mathbb{C}^{1}$ if and only if $p_{j}=t^{d_{j}-1}$, as in (b).

To check when $C \cap S \cong \mathbb{C}^{1}$, we argue in a similar way. We have $C \cdot\left(D+A_{1}+A_{2}\right)=4$ and $C$ meets $D$ at $L_{1, \infty} \cap L_{2, \infty}$, so $C \cap S \cong \mathbb{C}^{1}$ if and only if all the blowups in the decomposition of $\varphi$ touch the image of $C$. After the first blowup over $\ell_{j, 1} \cap \ell_{3-j, 0}$ the proper transform of $c$ meets the exceptional curve at a point of coordinate $\left(x_{3-j}-1\right) / x_{j}=1$, so all the remaining blowups touch it if and only if $p_{j}=1$ (i.e. there are no more blowups) or $p_{j}=t^{d_{j}-1}-t^{d_{j}-2}$, since as before the next infinitely near points will have coordinate zero on the exceptional curves. In this case, $C^{2}=c^{2}-\left(d_{1}+d_{2}\right)=2-d_{1}-d_{2}$, as in (c).

It remains to show that if $\Gamma \cap S \cong \mathbb{C}^{1}$ for some curve $\Gamma \subseteq X$ then $\Gamma$ equals $A_{j}, L_{1, j}$ or $C$. Consider first the case when $\Gamma$ is vertical for the $\mathbb{P}^{1}$-fibration induced by $\left|L_{j, \infty}\right|$, so $\Gamma \subseteq \varphi^{*} \ell_{j, t}$ for some $t \in \mathbb{C}^{1}$ because $\Gamma \nsubseteq D$. If $t \notin\{0,1\}$ then $\Gamma \cap S \cong \mathbb{C}^{*}$, which is false. If $t=0$ then $\Gamma=A_{j}$ because $\varphi^{*} \ell_{j, 0}=L_{j, 0}+T_{j}+A_{j}$. If $t=1$ then $\Gamma=L_{1, j}$ or $A_{3-j}$ because $\left(\varphi^{*} \ell_{j, 1}\right)_{\text {red }}=L_{j, 1}+T_{3-j}+A_{3-j}$.

Consider now the case when $\Gamma$ is horizontal for both $\mathbb{P}^{1}$-fibrations induced by $\left|L_{j, \infty}\right|, j \in\{1,2\}$. Then $\Gamma$ meets both $L_{j, \infty}$, so the point $\Gamma \cap D$ equals $L_{1, \infty} \cap L_{2, \infty}$. Since $\Gamma$ is disjoint from $L_{1,0}+L_{2,0}=[1,1]$, the linear system of the latter induces a $\mathbb{P}^{1}$-fibration for which $\Gamma$ is vertical. The curves $L_{j, \infty}$ are 1 -sections for this $\mathbb{P}^{1}$-fibration, so $\Gamma \cdot L_{j, \infty}=1$. It follows that $\varphi(\Gamma) \subseteq \mathbb{P}^{1} \times \mathbb{P}^{1}$ is of type $(1,1)$, passes through $(\infty, \infty)$, and because $\Gamma \cdot L_{j, 0}=0, \varphi(\Gamma)$ passes through the base point of $\varphi^{-1}$ on $\ell_{j, 0}$, that is, through $(0,1)$ for $j=1$ and $(1,0)$ for $j=2$. Thus $\varphi(\Gamma)=c$, as claimed.

Lemma 5.3. For any $\mathbb{C}^{*}$-fibration of $S$ one can find on $S$ two disjoint, vertical curves isomorphic to $\mathbb{C}^{1}$, whose closures $\Gamma_{1}, \Gamma_{2} \subseteq X$ satisfy $\Gamma_{i}^{2} \geqslant-1$, with strict inequality if $\Gamma_{i} \cdot D \geqslant 2$.

Proof. Fix a $\mathbb{C}^{*}$-fibration $f$ of $S$. Let $\alpha_{1}: X^{\prime \prime} \longrightarrow X$ be the minimal resolution of the base points of $f$ on $X$, and let $\alpha_{2}: X^{\prime \prime} \longrightarrow X^{\prime}$ be the contraction of all vertical $(-1)$-curves which are superfluous in the subsequent images of $\left(\alpha_{1}^{*} D\right)_{\text {red }}$. Put $\alpha=\alpha_{2} \circ \alpha_{1}^{-1}$ and $D^{\prime}=\left(\left(\alpha^{-1}\right)^{*} D\right)_{\text {red }}$.

Denote the circular subdivisor of $D$ by $R$. Then $\alpha_{1}^{*} R$ contains a unique circular subdivisor $R^{\prime \prime}$. Because all fibers are trees, $R^{\prime \prime}$ contains a horizontal component, say $H$. Suppose $R^{\prime \prime}-H$ is vertical. Then it is connected and meets $H$ twice, so $H$ is a 2-section, in fact $H=D_{\text {hor }}^{\prime \prime}$; a contradiction with Lemma 2.4. Therefore, $R^{\prime \prime}$ contains two 1-sections. In particular, $R_{\text {hor }}^{\prime \prime}=D_{\text {hor }}^{\prime \prime}$, so $\alpha_{1}$ is a composition of inner blowups with respect to the images of $R^{\prime \prime}$, hence it does not touch $\alpha_{1}^{*}(D-R)$. Because the latter contains no $(-1)$-curves and meets $R^{\prime \prime}$ in branching components of $D^{\prime \prime}, \alpha_{2}$ does not touch it, either. In other words, $\alpha$ is a composition of inner blowups on the images of $R^{\prime \prime}$ and does not touch the twigs of $D^{\prime \prime}$.

In particular, $D_{\text {hor }}^{\prime}$ consists of two 1-sections, both contained in $R^{\prime}=\left(\alpha_{2}\right)_{*} R^{\prime \prime}$.

Consider the case when $D_{\text {vert }}^{\prime}$ has a $(-1)$-curve, say $\Gamma$, and let $F$ be the fiber containing it. Because $D^{\prime}$ has no superfluous vertical $(-1)$-curves, $\beta_{D^{\prime}}(\Gamma) \geqslant 3$, so $\Gamma \subseteq R^{\prime}$ meets a maximal twig $T$ of $D^{\prime}$. Because $\alpha^{-1}$ does not touch $T$, we get $\alpha_{*}^{-1}(T+\Gamma)=T_{j}+L_{j, 0}=\left[(2)_{d_{j}}, 1\right]$ for some $j \in\{1,2\}$. Since $\beta_{F_{\text {red }}}(\Gamma) \leqslant 2$, $\Gamma$ meets $R_{\text {hor }}^{\prime}$. The latter consists of 1 -sections, so $\Gamma$ has multiplicity 1 in $F$, and thus $\Gamma$ is a tip of $F$. Because $F$ contracts to a 0 -curve, it follows that $F=\left[1,(2)_{d_{j}}, 1\right]$. The $(-1)$-curve $\Gamma^{\prime}=F-T-\Gamma$ satisfies 
$\Gamma^{\prime} \cdot D^{\prime}=1+(F-T-\Gamma) \cdot D_{\text {hor }}^{\prime}=1$, so $A=\alpha_{*}^{-1} \Gamma^{\prime}$ satisfies $A \cdot D=A \cdot T_{j}=1$, thus $A=A_{3-j}$ by Lemma 5.2. It follows that $F=\alpha^{*}\left(A_{j}+T_{j}+L_{3-j, 0}\right)$, so our $\mathbb{P}^{1}$-fibration is in fact induced by a pullback of $\left|L_{3-j, \infty}\right|$. Therefore, $\alpha=\mathrm{id}$ and the curves $A_{j} \cap S$ and $A_{3-j} \cap S$ are as required.

Consider the case when $D_{\text {vert }}^{\prime}$ contains no $(-1)$-curves. Because $R^{\prime}$ is circular, $R_{\text {hor }}^{\prime}$ has $2-b_{0}\left(R_{\text {vert }}^{\prime}\right) \in$ $\{0,1,2\}$ nodes. Let $F_{r}$ be a fiber passing through a node $r \in R_{\text {hor }}^{\prime}$. Then $\left(F_{r} \cdot D_{\text {hor }}^{\prime}\right)_{r}=2$, so because each connected component of $D_{\text {vert }}^{\prime}$ meets $D_{\text {hor }}^{\prime}, F_{r} \cap D^{\prime}=\{r\}$. Because $X^{\prime} \backslash D^{\prime} \cong S$ is affine, $F_{r} \cap\left(X^{\prime} \backslash D^{\prime}\right) \cong \mathbb{C}^{1}$. The curve $F_{r}$ meets each 1 -section in $D_{\text {hor }}^{\prime}$ once, so the above description of $\alpha$ shows that $\alpha_{2}$ touches $\left(\alpha_{2}^{-1}\right)_{*} F_{r}$ at most once. Therefore, $\left(\alpha_{*}^{-1} F_{r}\right)^{2} \geqslant F_{r}^{2}-1=-1$, and the equality holds if and only if the proper transform of $F_{r}$ is touched by $\alpha_{2}$ and not by $\alpha_{1}$, in which case $\alpha_{*}^{-1} F_{r} \cdot D=1$. Hence $F_{r} \cap S$ is as in the statement of the lemma.

Similarly, if $A \subseteq X^{\prime}$ is a vertical (-1)-curve such that $A \cdot D^{\prime}=1$, then $A \nsubseteq D^{\prime}$ by assumption, and $\alpha_{2}$ does not touch $\left(\alpha_{2}^{-1}\right)_{*} A$, hence $A \cap S$ is as in the statement, and clearly $A$ is disjoint from any $F_{r}$. Therefore, it remains to find $b_{0}\left(R_{\text {vert }}^{\prime}\right)$ disjoint vertical (-1)-curves $A \subseteq X^{\prime}$ such that $A \cdot D^{\prime}=1$.

Assume that every connected component $V$ of $R_{\text {vert }}^{\prime}$ is contained in a fiber $F_{V} \neq V$. Let $A_{V} \subseteq F_{V}$ be a $(-1)$-curve. Then $A_{V} \not \subset D^{\prime}, A_{V} \cdot D_{\text {hor }}^{\prime} \leqslant\left(F_{V}-V\right) \cdot D_{\text {hor }}^{\prime}=0$ and $A_{V} \cdot D_{\text {vert }}^{\prime}=1$ because $F_{V}$ is a tree, so $A_{V} \cdot D^{\prime}=1$. This ends the proof because $A_{V} \cap A_{V^{\prime}}=\emptyset$ for $V \neq V^{\prime}$.

Thus we can assume that $R_{\text {vert }}^{\prime}$ contains a fiber. We have $3 \leqslant \# R^{\prime} \leqslant \# D^{\prime}=\rho\left(X^{\prime}\right)$ by Lemma 2.1. Hence $X^{\prime}$ contains a degenerate fiber $F$. Let $A$ be a $(-1)$-curve in $F$, so $A \nsubseteq D^{\prime}$.

Suppose that $F \subseteq D_{\text {vert }}^{\prime}+A$. Then $A$ is a unique $(-1)$-curve in $F$, so it has multiplicity $\mu \geqslant 2$ in $F$. Denote our $\mathbb{C}^{*}$-fibration of $S$ by $f$. Because $D^{\prime}$ contains a fiber, $f(S) \subseteq \mathbb{C}^{1}$, and we can assume that $f(A \cap S)=\{0\}$. Because $\mathbb{C}[S]$ is a UFD, $\left.A\right|_{S}$ is a divisor of a regular function, say $g$. Then $f / g^{\mu} \in \mathbb{C}[S]^{*}=\mathbb{C}^{*}$, so $f=\lambda g^{\mu}$ for some $\lambda \in \mathbb{C}^{*}$. A general fiber of such $f$ is reducible, a contradiction.

Suppose $L \cdot D^{\prime} \geqslant 2$ for every $(-1)$-curve $L \subseteq F$. Then $2 \leqslant L \cdot D^{\prime} \leqslant b_{0}\left(F \cap D^{\prime}\right)$ because $F$ is a tree, and $b_{0}\left(F \cap D^{\prime}\right) \leqslant F \cdot D_{\text {hor }}^{\prime}=2$ because $D^{\prime}$ is connected. It follows that $A$ is a unique (-1)-curve in $F$, and meets two connected components of $F \cap D^{\prime}$. Because $F$ meets $D_{\text {hor }}^{\prime}$ in components of multiplicity 1 , it follows that $F$ is a chain meeting $D^{\prime}$ in tips contained in $D_{\text {vert }}^{\prime}$. Hence $F \subseteq D_{\text {vert }}^{\prime}+A$; a contradiction.

Thus we can assume $A \cdot D^{\prime}=1$. If $b_{0}\left(R_{\text {vert }}^{\prime}\right)<2$ then the lemma follows, so we can assume $b_{0}\left(R_{\text {vert }}^{\prime}\right)=2$. Lemma 2.1(b) implies that $D^{\prime}$ contains exactly one fiber, so some connected component $V$ of $R_{\text {vert }}^{\prime}$ is contained in a degenerate fiber $F$ as above. Recall that $A \subseteq F$ is a (-1)-curve such that $A \nsubseteq D^{\prime}$, $A \cdot D^{\prime}=1$ and $F \nsubseteq D_{\text {vert }}^{\prime}+A$.

For every component $L$ of $F-D_{\text {vert }}^{\prime}$ we have $L \cdot D_{\text {hor }}^{\prime} \leqslant\left(F^{\prime}-V\right) \cdot D_{\text {hor }}^{\prime}=0$, so since $F$ is a tree, $L \cdot D^{\prime}=1$ and all such $L$ 's are disjoint. If two of them are $(-1)$-curves then the lemma follows. Hence we can assume that $A$ is a unique $(-1)$-curve in $F$. Put $T=0$ if $A$ meets $V$, otherwise denote by $T$ the maximal twig of $D^{\prime}$ meeting $A$. If $T \neq 0$ then $\alpha^{-1}$ does not touch $A+T$, hence by Lemma 5.2, $A+T=\alpha_{*}\left(A_{j}+T_{j}\right)=\left[1,(2)_{d_{j}}\right]$ for some $j \in\{1,2\}$. Moreover, if $T$ meets a curve $L \not D^{\prime}$ with $L \cdot D^{\prime}=1$ then Lemma 5.2 gives $L=A$. Hence $T \cdot\left(F_{\text {red }}-A-V\right)=0$. In any case, let $\tau$ be the contraction of $A+T$. Then $\tau$ does not touch $F_{\text {red }}-V$, and the fiber $\tau_{*} F$ has a unique $(-1)$-curve, contained in $\tau_{*} V$. Perform further inner contractions with respect to the images of the chain $\tau_{*} V$ until one of the components of the image of $F$ meeting a 1-section in the image of $D_{\text {hor }}^{\prime}$ becomes a $(-1)$-curve. Then the latter has multiplicity 1 in the image of $F$, so it is not its unique $(-1)$-curve. It follows that the image of $F$ is of type $[1,1]$ and both its components are in the image of $V$. Hence $F \subseteq D_{\text {vert }}^{\prime}+A$; a contradiction.

In the following, we fix coordinates on $S=S_{p_{1}, p_{2}}$, treating it as a closed subset of $\mathbb{C}^{4}$ given by (5.1).

Lemma 5.4 (Open tori in $S$ ). Fix an open subset $U \subseteq S$ and a ring homomorphism $\sigma: \mathbb{C}\left[x_{1}, x_{2}, y_{1}, y_{2}\right] \longrightarrow$ $\mathbb{C}\left[v_{1}^{ \pm 1}, v_{2}^{ \pm 1}\right]$ as in (a)-(c) below. Then $\sigma$ induces an isomorphism $U \cong \mathbb{C}^{*} \times \mathbb{C}^{*}$.

(a) $U=S \backslash\left(A_{1}+A_{2}\right)$ and $\sigma\left(x_{j}\right)=v_{j}, \sigma\left(y_{j}\right)=\left(v_{3-j}-\widehat{p}_{j}\left(v_{j}\right)\right) v_{j}^{-d_{j}}$ for $j \in\{1,2\}$;

(b) In case $p_{3-j}=1$ for some $j \in\{1,2\}: U=S \backslash\left(A_{j}+L_{j, 1}\right)$ and

$$
\sigma\left(x_{j}\right)=v_{j}, \quad \sigma\left(x_{3-j}\right)=\left(v_{j}-1\right) v_{3-j}^{-1}, \quad \sigma\left(y_{j}\right)=\left(v_{j}-1-\widehat{p}_{j}\left(v_{j}\right) v_{3-j}\right) \cdot\left(v_{j}^{d_{j}} v_{3-j}\right)^{-1}, \quad \sigma\left(y_{3-j}\right)=v_{3-j} ;
$$

(c) In case $p_{1}=p_{2}=1: U=S \backslash\left(C+L_{j, 1}\right)$ for some $j \in\{1,2\}$ and

$$
\sigma\left(x_{j}\right)=-\left(v_{2}+1\right) v_{1}^{-1}, \quad \sigma\left(x_{3-j}\right)=-\left(v_{1}+v_{2}+1\right) \cdot\left(v_{1} v_{2}\right)^{-1}, \quad \sigma\left(y_{j}\right)=\left(v_{1}+1\right) v_{2}^{-1}, \quad \sigma\left(y_{3-j}\right)=v_{2} .
$$

The volume form $\frac{d v_{1}}{v_{1}} \wedge \frac{d v_{2}}{v_{2}}$ on $U$ extends, up to a sign, to a volume form $\frac{d x_{1}}{x_{1}} \wedge \frac{d x_{2}}{x_{2}}$.

Proof. We check that by a direct computation that $\sigma$ is zero on the ideal of $S$, so it gives a morphism $\mathbb{C}^{*} \times \mathbb{C}^{*} \longrightarrow S$. It has a rational inverse, given by

$$
\text { (a) } v_{j} \mapsto x_{j}, j \in\{1,2\} ; \quad \text { (b) } v_{j} \mapsto x_{j}, v_{3-j} \mapsto y_{3-j} ; \quad \text { (c) } v_{1} \mapsto y_{1} y_{2}-1, v_{2} \mapsto y_{3-j} \text {. }
$$


This inverse is regular on the complement of:

(a) $\left\{x_{1} x_{2}=0\right\}=\left.\left(A_{1}+A_{2}\right)\right|_{S} ; \quad$ (b) $\left\{x_{j} y_{3-j}=0\right\}=\left.\left(A_{j}+L_{j, 1}\right)\right|_{S} ; \quad$ (c) $\left\{y_{3-j}\left(1-y_{1} y_{2}\right)=0\right\}=\left.\left(L_{j, 1}+C\right)\right|_{S}$; and sends $\frac{d v_{1}}{v_{1}} \wedge \frac{d v_{2}}{v_{2}}$ to $\pm \frac{d x_{1}}{x_{1}} \wedge \frac{d x_{2}}{x_{2}}$. This proves the lemma.

Remark. The formulas $\left\{y_{3-j}=0\right\}=\left.L_{j, 1}\right|_{S},\left\{y_{1} y_{2}=1\right\}=\left.C\right|_{S}$ used in the above proof do not hold for general $p_{1}, p_{2}$, but they do hold under the assumptions of (b) and (c), respectively.

Proposition 5.5 (Complete algebraic vector fields on $S_{p_{1}, p_{2}}$, cf. [KK11, Theorem 19]).

(a) Every $\mathbb{C}^{*}$-fibration of $S$ restricts to a $\mathbb{C}^{*}$-fibration of one of the open tori $U \subseteq S$ from Lemma 5.4

(b) For any $U \subseteq S$ from Lemma 5.4 and any $a_{j} \geqslant d_{j}, j \in\{1,2\}$, the complete vector field $\nu_{a_{1}, a_{2}}=$ $v_{1}^{a_{1}} v_{2}^{a_{2}}\left(a_{2} v_{1} \frac{\partial}{\partial v_{1}}-a_{1} v_{2} \frac{\partial}{\partial v_{2}}\right)$ on $U$ extends to a complete vector field on $S$.

(c) Every complete algebraic vector field on $S$ restricts to a complete vector field on some torus $U \subseteq S$ from Lemma 5.4. In particular, it has divergence zero with respect to the volume form $\frac{d x_{1}}{x_{1}} \wedge \frac{d x_{2}}{x_{2}}$.

(d) For each open torus $U \subseteq S$ from Lemma 5.4, the group $\operatorname{AAuthol}_{\text {hol }}(S) \cap \operatorname{AAuthol}_{\text {hol }}(U)$ acts on $U m$ transitively for every $m$.

Proof. (a) Fix a $\mathbb{C}^{*}$-fibration $p$ of $S$ and let $\Gamma_{1}, \Gamma_{2} \subseteq X$ be as in Lemma 5.3. Then $p$ restricts to a $\mathbb{C}^{*}$-fibration of $U=S \backslash\left(\Gamma_{1}+\Gamma_{2}\right)$. We claim that $U$ is as in Lemma 5.4. Because for $i \in\{1,2\} \Gamma_{i} \cap S \cong \mathbb{C}^{1}$, $\Gamma_{i}$ is one of the curves from Lemma 5.2. If $\left\{\Gamma_{1}, \Gamma_{2}\right\}=\left\{A_{1}, A_{2}\right\}$ then $U$ is as in Lemma 5.4(a). Assume that $\Gamma_{1}=C$. Because $C \cdot D=2$, by Lemma $5.30 \leqslant \Gamma_{1}^{2}=2-d_{1}-d_{2}$, so $d_{1}=d_{2}=1$, i.e. $p_{1}=p_{2}=1$. In this case, $C \cap S$ meets $A_{j} \cap S$ for both $j \in\{1,2\}$, so $\Gamma_{2}=L_{1, j}$ for some $j \in\{1,2\}$ and therefore $U$ is as in Lemma 5.4(c). We are left with the case when $\Gamma_{1}=L_{1, j}$ for some $j \in\{1,2\}$ and $\Gamma_{2} \neq C$. As before, the condition $\Gamma_{1}^{2} \geqslant-1$ from Lemma 5.3 implies by Lemma $5.2(\mathrm{~b})$ that $d_{j}=1$, i. e. $p_{j}=1$. In this case, $L_{1, j} \cap S$ meets $A_{3-j} \cap S$ and $L_{3-j, 1} \cap S$, so $\Gamma_{2}=A_{j}$ or $C$. The second case is excluded by assumption, so the first case holds and $U$ is as in Lemma 5.4(b).

(b) follows from a direct computation, see [And00, Corollary 2].

(c) Part (b) implies that complete algebraic vector fields on $S$ do not share a rational first integral. Hence by [KKL20, Theorem 1.6], for any complete algebraic vector field $\xi$ on $S$ there is a $\mathbb{C}^{1}$ - or $\mathbb{C}^{*}$-fibration of $S$ preserved by the flow of $\xi$. Because $\kappa(S)=0, S$ admits no $\mathbb{C}^{1}$-fibrations, hence $\xi$ preserves one of the $\mathbb{C}^{*}$-fibrations from (a). In each case, the complement of $U$ is a union of vertical curves isomorphic to $\mathbb{C}^{1}$, so the flow of $\xi$ cannot send them to non-degenerate fibers in $U$. It follows that $\xi$ restricts to a complete vector field on $U$. In particular, by [And00, Corollary 1] it preserves the volume form $\frac{d v_{1}}{v_{1}} \wedge \frac{d v_{2}}{v_{2}}$, where $v_{1}, v_{2}$ are coordinates on $U \cong \mathbb{C}^{*} \times \mathbb{C}^{*}$. Lemma 5.4 implies that $\xi$ preserves $\frac{d x_{1}}{x_{1}} \wedge \frac{d x_{2}}{x_{2}}$, too.

(d) Choose integers $a_{i j}, i, j \in\{1,2\}$ such that $a_{i j} \geqslant d_{j}$ and $\operatorname{det}\left[a_{i j}\right]=1$, and put $\sigma_{i}=\nu_{a_{i 1}, a_{i 2}}$, $f_{i}=v_{1}^{a_{i 1}} v_{2}^{a_{i 2}} \in \operatorname{ker} \sigma_{i} \backslash \operatorname{ker} \sigma_{3-i}$. Now the result follows from [KK11, Propostion 8.9]. Indeed, although that result is formulated in loc. cit. for $G=\operatorname{AAuthol}_{\text {hol }}(U)$, the proof actually shows $m$-transitivity for the group generated by the elements of the flows of $q\left(f_{i}\right) \cdot \sigma_{i}$ for $q \in \mathbb{C}[t]$.

Proof of Theorem 1.6. Part (a) was shown in Corollary 2.6(c). For (b), note that the open subset $S \backslash Z$ of $S$ is precisely the union of all $U \subseteq S$ from Lemma 5.4. By Proposition 5.5(c), $S \backslash Z$ is fixed by AAut $_{\text {hol }}(S)$. On the other hand, by Proposition 5.5(d) each $U \subseteq S$ from Lemma 5.4 is contained in a single orbit of AAuthol $(S)$. Because every two such $U$, being open and dense in $S$, have nonempty intersection, they are all contained in the same orbit of $\mathrm{AAut}_{\text {hol }}(S)$, which therefore equals $S \backslash Z$. Proposition 5.5(d) implies that $\mathrm{AAut}_{\mathrm{hol}}(S)$ acts on this orbit $m$-transitively for every $m$, as claimed.

Remark 5.6 (Density property for $S_{p_{1}, p_{2}}$ ). In view of Theorem 1.6(b), it is natural to ask for an even stronger property than $m$-transitivity of $\operatorname{AAut}_{\text {hol }}(S)$, namely for the algebraic density property (ADP), see [KK11]. Recall that a smooth affine variety $X$ has $\mathrm{ADP}$ if the Lie algebra $\operatorname{VF}_{\text {alg }}(X)$ of algebraic vector fields on $X$ coincides with the Lie algebra $\operatorname{Lie}_{a l g}(X)$ generated by complete ones. Since in our case all vector fields preserve $Z$, one should rather ask if the relative ADP holds, see [KLL15], namely, if there exists $l \geqslant 0$ such that $I^{l} \mathrm{VF}_{\text {alg }}(S) \subseteq \operatorname{Lie}_{\text {alg }}(S)$, where $I$ is the ideal of $Z$. This, however, is not true since by Lemma $5.4(\mathrm{c})$ all elements of $\operatorname{Lie}_{\text {alg }}(S)$ preserve the volume form $\omega=\frac{d x_{1}}{x_{1}} \wedge \frac{d x_{2}}{x_{2}}$. Nonetheless, one could ask for the relative volume density property, defined as follows (see [KK16]). Let $X$ be a normal variety with a volume form $\omega$, and let $Y \subseteq X$ be a closed subset containing Sing $X$. Denote by $\operatorname{VF}_{\text {alg }}^{\omega}(X, Y)$ the Lie algebra of those algebraic vector fields on $X$ which vanish on $Y$ and preserve the volume form $\omega$; and by $\operatorname{Lie}_{\text {alg }}^{\omega}(X, Y)$ the Lie algebra generated by complete vector fields in $\operatorname{VF}_{\text {alg }}^{\omega}(X, Y)$. We say that $X$ has an algebraic volume density property (AVDP) relative to $Y$ if there exists $l \geqslant 0$ such that $I^{l} \mathrm{VF}_{\text {alg }}^{\omega}(X, Y) \subseteq \operatorname{Lie}_{\text {alg }}^{\omega}(X, Y)$, where $I$ is the ideal of $Y$. In this setting, one could ask the following: 
Question. Let $Z \subseteq S_{p_{1}, p_{2}}$ be as in Theorem 1.6(b). Does $S_{p_{1}, p_{2}}$ have AVDP relative to $Z$ ?

For $p_{1}=p_{2}=1$ (so $Z=\emptyset$ ), the answer to this question is positive [KK16, Theorem 6].

\section{REFERENCES}

[AFW15] M. Aschenbrenner, S. Friedl, and H. Wilton, 3-manifold groups, EMS Series of Lectures in Mathematics, European Mathematical Society (EMS), Zürich, 2015.

[And00] E. Andersén, Complete vector fields on $\left(\mathbf{C}^{*}\right)^{n}$, Proc. Amer. Math. Soc. 128 (2000), no. 4, $1079-1085$.

[Bro67] R. Brown, Groupoids and van Kampen's theorem, Proc. London Math. Soc. (3) 17 (1967), $385-401$.

[BW01] M. Brittenham and Y. Q. Wu, The classification of exceptional Dehn surgeries on 2-bridge knots, Comm. Anal. Geom. 9 (2001), no. 1, 97-113.

[Eis95] D. Eisenbud, Commutative algebra, with a view toward algebraic geometry, Graduate Texts in Mathematics, vol. 150, Springer-Verlag, New York, 1995.

[EN85] D. Eisenbud and W. Neumann, Three-dimensional link theory and invariants of plane curve singularities, Annals of Mathematics Studies, vol. 110, Princeton University Press, Princeton, NJ, 1985.

[FKN19] G. Freudenburg, H. Kojima, and T. Nagamine, Smooth factorial affine surfaces of logarithmic Kodaira dimension zero with trivial units, arXiv:1910.03494, 2019.

[FKZ07] H. Flenner, S. Kaliman, and M. Zaidenberg, Birational transformations of weighted graphs, Affine algebraic geometry, Osaka Univ. Press, Osaka, 2007, pp. 107-147.

[Fuj82] T. Fujita, On the topology of noncomplete algebraic surfaces, J. Fac. Sci. Univ. Tokyo Sect. IA Math. 29 (1982), no. 3, 503-566.

[FZ94] H. Flenner and M. Zaidenberg, $\mathbb{Q}$-acyclic surfaces and their deformations, Classification of algebraic varieties (L'Aquila, 1992), Contemp. Math., vol. 162, Amer. Math. Soc., Providence, RI, 1994, pp. 143-208.

[GM88] R. V. Gurjar and M. Miyanishi, Affine surfaces with $\bar{\kappa} \leqslant 1$, Algebraic geometry and commutative algebra, Vol. I, Kinokuniya, Tokyo, 1988, pp. 99-124.

[GS99] R. Gompf and A. Stipsicz, 4-manifolds and Kirby calculus, Graduate Studies in Mathematics, vol. 20, American Mathematical Society, Providence, RI, 1999.

[Har77] R. Hartshorne, Algebraic geometry, Springer-Verlag, New York, 1977, Graduate Texts in Mathematics, 52.

[Hat07] A. Hatcher, Notes on basic 3-manifold topology, pi.math.cornell.edu/ hatcher/3M/3Mfds.pdf, 2007.

[HT85] A. Hatcher and W. Thurston, Incompressible surfaces in 2-bridge knot complements, Invent. Math. 79 (1985), no. $2,225-246$.

[IF77] S. Iitaka and T. Fujita, Cancellation theorem for algebraic varieties, J. Fac. Sci. Univ. Tokyo Sect. IA Math. 24 (1977), no. 1, 123-127.

[Iit82] S. Iitaka, Algebraic geometry, Graduate Texts in Mathematics, vol. 76, Springer-Verlag, New York, 1982, An introduction to birational geometry of algebraic varieties, North-Holland Mathematical Library, 24.

[Kal94] S. Kaliman, Exotic analytic structures and Eisenman intrinsic measures, Israel J. Math. 88 (1994), no. 1-3, 411423.

[Kaw79] Y. Kawamata, On the classification of noncomplete algebraic surfaces, Algebraic geometry (Proc. Summer Meeting, Univ. Copenhagen, Copenhagen, 1978), Lecture Notes in Math., vol. 732, Springer, Berlin, 1979, pp. $215-232$.

[KK11] S. Kaliman and F. Kutzschebauch, On the present state of the Andersén-Lempert theory, Affine algebraic geometry, CRM Proc. Lecture Notes, vol. 54, Amer. Math. Soc., Providence, RI, 2011, pp. 85-122.

[KK16] On algebraic volume density property, Transform. Groups 21 (2016), no. 2, 451-478.

[KKL20] S. Kaliman, F. Kutzschebauch, and M. Leuenberger, Complete algebraic vector fields on affine surfaces, Internat. J. Math. 31 (2020), no. 3, 2050018, 50.

[KLL15] F. Kutzschebauch, M. Leuenberger, and A. Liendo, The algebraic density property for affine toric varieties, J. Pure Appl. Algebra 219 (2015), no. 8, 3685-3700.

[Koj99] H. Kojima, Open rational surfaces with logarithmic Kodaira dimension zero, Internat. J. Math. 10 (1999), no. 5, 619-642.

[Miy01] M. Miyanishi, Open algebraic surfaces, CRM Monograph Series, vol. 12, American Mathematical Society, Providence, RI, 2001.

[Mos71] L. Moser, Elementary surgery along a torus knot, Pacific J. Math. 38 (1971), 737-745.

[Mum61] D. Mumford, The topology of normal singularities of an algebraic surface and a criterion for simplicity, Inst. Hautes Études Sci. Publ. Math. (1961), no. 9, 5-22.

[Neu81] W. Neumann, A calculus for plumbing applied to the topology of complex surface singularities and degenerating complex curves, Trans. Amer. Math. Soc. 268 (1981), no. 2, 299-344.

[Pal19] K. Palka, Cuspidal curves, minimal models and Zaidenberg's finiteness conjecture, J. Reine Angew. Math. 747 (2019), 147-174.

[Prz83] J. Przytycki, Incompressibility of surfaces after Dehn surgery, Michigan Math. J. 30 (1983), no. 3, $289-308$.

Basque Center for Applied Mathematics, Alameda de Mazarredo 14, 48009 Bilbao, Spain

Email address: tpelka@bcamath.org

Instytut Matematyki, Uniwersytet Jagielloński, ul. Łojasiewicza 6, 30-348 Kraków, Poland

Email address: pawel.razny@uj.edu.pl 